\title{
Equalization Techniques for Distributed Space-Time Block Codes With Amplify-and-Forward Relaying
}

\author{
Hakam Mheidat, Member, IEEE, Murat Uysal, Member, IEEE, and Naofal Al-Dhahir, Senior Member, IEEE
}

\begin{abstract}
In this paper, we investigate equalization methods for cooperative diversity schemes over frequency-selective fading channels. Specifically, we consider three equalization schemes proposed originally for conventional space-time block codes (STBC) [1]-[3] and extend them to distributed STBC in a cooperative transmission scenario with amplify-and-forward relaying. The distributed STBC equalization schemes are named after their original counterparts as distributed time-reversal (D-TR) STBC, distributed single-carrier (D-SC) STBC, and distributed orthogonal frequency division multiplexed (D-OFDM) STBC. The underlying orthogonality of distributed STBC results in decoupled data streams at the receiver side allowing low-complexity implementations. Without loss of generality, we consider a single-relay scenario where the source-to-relay $S \rightarrow R$, relay-to-destination $R \rightarrow D$, and source-to-destination $S \rightarrow D$ links experience possibly different channel delay spreads. Under the assumption of perfect power control for the relay terminal and high signal-to-noise ratio (SNR) for the underlying links, our performance analysis demonstrates that D-TR-STBC, D-SC-STBC, and coded D-OFDM-STBC schemes are able to achieve a maximum diversity order of $\min \left(L_{1}, L_{3}\right)+L_{2}+2$ where $L_{1}, L_{2}$, and $L_{3}$ are the channel memory lengths for $S \rightarrow R, S \rightarrow D$, and $R \rightarrow D$ links, respectively. This illustrates that the minimum of the multipath diversity orders experienced in $S \rightarrow R$ and $R \rightarrow D$ links becomes the performance bottleneck for the relaying path. For the case of a nonfading relaying path where line-of-sight propagation is possible in either one of these underlying links, we demonstrate that diversity orders of $L_{1}+L_{2}+2$ and $L_{3}+L_{2}+2$ are achievable assuming nonfading $S \rightarrow R$ and $R \rightarrow D$ links, respectively. An extensive Monte Carlo simulation study is presented to corroborate the analytical results and to provide detailed performance comparisons among the three candidate equalization schemes.
\end{abstract}

Index Terms-Cooperative diversity, distributed space-time block coding (STBC), equalization, fading channels, pairwise error probability.

Manuscript received August 2, 2005; revised April 25, 2006. The work of M. Uysal was supported in part by an NSERC Special Opportunity (Grant SROPJ305821-05). The work of N. Al-Dhahir was supported in part by the Texas Advanced Technology (ATP) program under Contract 009741-0023-2003 and by NSF Contracts CCF 0430654 and DMS 0528010. The associate editor coordinating the review of this paper and approving it for publication was Dr. Michael Buehrer.

H. Mheidat and M. Uysal are with the Department of Electrical and Computer Engineering, University of Waterloo, ON N2G3L1, Canada (e-mail: hmheidat@ece.uwaterloo.ca; muysal@ece.uwaterloo.ca).

N. Al-Dhahir is with the Department of Electrical Engineering, The University of Texas at Dallas, Richardson, TX 75083 USA (e-mail: aldhahir@utdallas.edu).

Color versions of one or more of the figures in this paper are available online at http://ieeexplore.ieee.org.

Digital Object Identifier 10.1109/TSP.2006.889974

\section{INTRODUCTION}

$\mathbf{T}$ HE increasing demand for wireless multimedia and interactive internet services is fueling intensive research efforts on higher-speed data transmission and improved power efficiency compared to current wireless communication systems. Spatial diversity offers significant improvement in link reliability and spectral efficiency through the use of multiple antennas at the transmitter and/or receiver side [4]-[7]. These gains are typically realized at the physical layer and require colocated antenna elements (i.e., physical antenna arrays) at the base station and/or the mobile terminal. Multiple-antenna techniques are very attractive for deployment in cellular applications at base stations and have already been included in the third-generation wireless standards. Unfortunately, the use of multiple antennas might not be practical at the cellular mobile devices, as well as in ad hoc mobile networks due to size and power constraints. Recently, it has been demonstrated that "cooperative diversity," also known as "user cooperation," provides an effective means of improving spectral and power efficiency of wireless networks as an alternative to multiple-antenna transmission schemes [8]-[11]. The main idea behind cooperative diversity is based on the observation that in a wireless environment, the signal transmitted by the source nodes is overheard by other nodes, which can be defined as partners. The source and its partners can jointly process and transmit their information, creating a "virtual antenna array" although each of them is equipped with only one antenna.

The pioneering works on cooperative diversity address information-theoretic aspects of cooperative networks investigating achievable rate regions and outage probabilities. The outage analysis in [10] relies on a random coding argument and demonstrates that full spatial diversity can be achieved using such a rich set of codes. Laneman et al. [10] suggest the use of "conventional" orthogonal space-time block coding (STBC) in a "distributed" fashion for practical implementation of user cooperation. Nabar et al. [12], [13] analyze distributed STBC operating in an amplify-and-forward (AF) mode through the derivation of pairwise error probability (PEP) expressions. They show that the original design criteria for conventional STBC (i.e., rank and determinant criteria) still apply for the design of distributed STBC schemes under the assumption that appropriate power control rules are employed at relays.

Since space-time codes (conventional ones as well as their distributed versions) were originally developed for frequency-flat channels, applying them over frequency-selective channels becomes a challenging design problem. The dispersive nature of such channels causes intersymbol interference (ISI), 
leading to unavoidable performance degradation. A variety of equalization techniques, which were initially developed for single-input single-output (SISO) systems can be applied to distributed STBC. However, straightforward extensions result in an excessive complexity especially for higher-order modulation schemes and/or long channel memory [14]. Among the equalization techniques studied for STBC, three of those deserve particular attention due to their low-complexity, namely time-reversal STBC (TR-STBC) [1], single-carrier frequency-domain equalization for STBC (SC-STBC) [2], and orthogonal frequency division multiplexed (OFDM)-STBC [3]. An overview and comparison of these schemes can be found in [15]-[17]. In this paper, we extend the three aforementioned equalization schemes to a relay-assisted transmission scenario, carefully exploiting the underlying orthogonality of distributed STBC. We present a thorough comparative performance analysis of these schemes through the derivation of PEP expressions and an extensive Monte Carlo simulation study.

Related Work and Contributions: Most of the current literature on cooperative diversity assumes frequency-flat fading channel. Along with the conference version of this paper [18], there have been only a few sporadic results reported on broadband cooperative transmission techniques for frequency-selective channels. Yatawatta et al. [19] study an OFDM cooperative diversity system assuming AF relaying and derive upper bounds on the channel capacity. They also investigate the achievable diversity order for distributed cooperative OFDM assuming a nonfading interuser channel. Barbarossa et al. [20] investigate the performance of a distributed OFDM-STBC scheme through a simulation study considering both $\mathrm{AF}$ and decode-and-forward (DF) relaying. Building upon their previous work on distributed STBC [21], Anghel et al. [22] study the performance of a relay-assisted uplink OFDM-STBC scheme and derive an expression for symbol error probability assuming DF with no error propagation. To the best of our knowledge, the conference version of this current paper [18] is the first attempt to investigate TR-STBC and SC-STBC in a relay-assisted transmission scenario (as well as OFDM-STBC along with the aforementioned papers [19], [20], [22]). We should further emphasize that our transmission model builds upon the Alamouti code (i.e., STBC for two transmit antennas) although extensions to other STBCs are straightforward, but not pursued here due to space limitations. Our contributions in this work are summarized as follows.

- We propose three broadband cooperative transmission techniques for distributed STBC. The proposed schemes, so-called distributed time-reversal (D-TR)-STBC, distributed single-carrier (D-SC)-STBC, and D-OFDM-STBC, are extensions of conventional STBC equalization schemes which are able to preserve low-decoding complexity due to the underlying orthogonality of distributed STBC.

- We derive PEP expressions for D-TR-STBC and D-SC-STBC schemes over frequency-selective channels. Through the derived PEPs, we demonstrate that both schemes are able to achieve a maximum diversity order of $\min \left(L_{1}, L_{3}\right)+L_{2}+2$ where $L_{1}, L_{2}$, and $L_{3}$ are the channel memory lengths for source-to-relay $(S \rightarrow R)$, source-to-destination $(S \rightarrow D)$, and relay-to-destination
$(R \rightarrow D)$ links, respectively. This illustrates that the smaller multipath diversity order experienced in $S \rightarrow R$ and $R \rightarrow D$ links becomes performance limiting for the relaying path.

- We derive PEP expressions for uncoded D-OFDM-STBC and frequency-interleaved D-OFDM-STBC concatenated with TCM (trellis coded modulation). Uncoded D-OFDM-STBC is not able to exploit multipath diversity and achieves only a diversity order of two in a single-relay scenario. The achievable diversity order for D-OFDM-TCM-STBC is given by $\min \left(\lceil\mathrm{ECL} / 2\rceil, L_{3}, L_{1}\right)+\min \left(\lceil\mathrm{ECL} / 2\rceil, L_{2}\right)+2$ where $\lceil\cdot\rceil$ denotes the ceiling function and ECL stands for the effective code length of the outer trellis code. Our derivation clearly points out that with an appropriate design of an outer TCM code, i.e., with sufficiently large ECL, coded D-OFDM-STBC achieves the same diversity order as D-TR-STBC and D-SC-STBC.

- Finally, a comprehensive Monte Carlo simulation study is presented to confirm the analytical observations and to further investigate several practical issues within the considered relay-assisted transmission scenario.

The rest of this paper is organized as follows: In Section II, the relay-assisted transmission model is introduced. The three equalization schemes under consideration for distributed STBC are described in Sections III, IV and V, followed by the corresponding diversity gain analysis in each section. Numerical results are presented in Section VI and the paper is concluded in Section VII. The Appendix provides mathematical details on the PEP derivations.

Notation: $\overline{(\cdot)},(\cdot)^{\mathrm{T}}$, and $(\cdot)^{\mathrm{H}}$ denote conjugate, transpose, and Hermitian transpose operations, respectively. $\otimes$ denotes Kronecker product, $\mathrm{E}[\cdot]$ denotes expectation, $\operatorname{tr}\{\cdot\}$ denotes a trace of a matrix, $[\cdot]_{k, l}$ denotes the $(k, l)^{t h}$ entry of a matrix, $[\cdot]_{k}$ denotes the $k^{t h}$ entry of a vector, $\|\cdot\|$ denotes the Euclidean norm of a vector, $*$ denotes convolution, $\mathbf{I}_{N}$ denotes the identity matrix of size $N, \mathbf{0}_{M \times M}$ denotes all-zero matrix of size $M \times M, \operatorname{diag}(\mathbf{v})$ stands for a diagonal matrix with $\mathbf{v}$ on its diagonal, and $\mathbf{Q}$ represents the $N \times N$ FFT matrix whose $(l, k)$ element is given by $\mathbf{Q}(l, k)=(1 / \sqrt{N}) \exp (-j 2 \pi l k / N)$ where $0 \leq l, k \leq N-1$. We define a set of $N \times N$ permutation matrices $\left\{\mathbf{P}_{N}^{\bar{q}}\right\}_{q=0}^{N-1}$ that performs a reversed cyclic shift, i.e., for a vector $\mathbf{a}=\left[\begin{array}{lll}a_{0} & \cdots & a_{N-1}\end{array}\right]^{\mathrm{T}},\left[\mathrm{P}_{N}^{q} \mathbf{a}\right]_{s}=\mathbf{a}((N-s+q) \bmod N)$. Bold upper-case letters denote matrices and bold lower-case letters denote vectors.

\section{TRANSMISSION MODEL}

A wireless communication scenario is considered where the source terminal $\mathrm{S}$ transmits information to the destination terminal $\mathrm{D}$ with the assistance of a relay terminal R (see Fig. 1). The channel impulse responses (CIRs) for $S \rightarrow R, S \rightarrow D$, and $R \rightarrow D$ links for the $j$ th transmission block are given by $\mathbf{h}_{1}^{j}=$ $\left[h_{S R}^{j}(0), \ldots, h_{S R}^{j}\left(L_{1}\right)\right]^{\mathrm{T}}, \mathbf{h}_{2}^{j}=\left[h_{S D}^{j}(0), \ldots, h_{S D}^{j}\left(L_{2}\right)\right]^{\mathrm{T}}$, and $\mathbf{h}_{3}^{j}=\left[h_{R D}^{j}(0), \ldots, h_{R D}^{j}\left(L_{3}\right)\right]^{\mathrm{T}}$, respectively, where $L_{1}, L_{2}$, and $L_{3}$ denote the corresponding channel memory lengths. The random vectors $\mathbf{h}_{1}^{j}, \mathbf{h}_{2}^{j}$, and $\mathbf{h}_{3}^{j}$ are assumed to be independent zero-mean complex Gaussian with power delay profile vectors denoted by $\mathbf{v}_{1}=\left[\sigma_{1}^{2}(0), \ldots, \sigma_{1}^{2}\left(L_{1}\right)\right]$, 


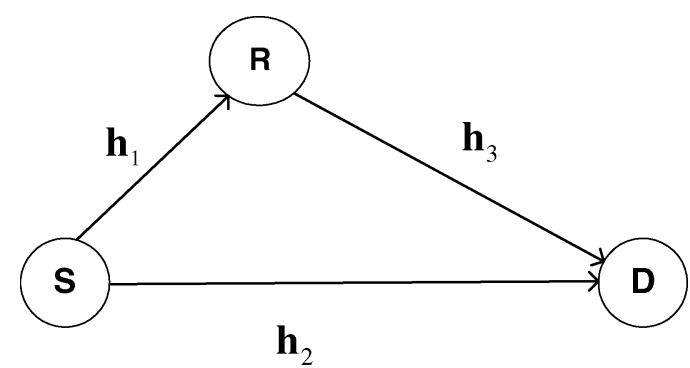

Fig. 1. Schematic representation of relay-assisted transmission.

$\mathbf{v}_{2}=\left[\sigma_{2}^{2}(0), \ldots, \sigma_{2}^{2}\left(L_{2}\right)\right]$, and $\mathbf{v}_{3}=\left[\sigma_{3}^{2}(0), \ldots, \sigma_{3}^{2}\left(L_{3}\right)\right]$, respectively, and are normalized such that $\sum_{l_{i}=0}^{L_{i}} \sigma_{i}^{2}\left(l_{i}\right)=1$, $i=1,2,3$. The CIRs are assumed to be constant over four consecutive blocks and vary independently every four blocks.

As for the user cooperation protocol, we adopt the so-called Protocol III proposed by Nabar et al. [12], [13]: Specifically, the source terminal communicates with the relay terminal during the first signaling interval. There is no transmission from source-to-destination during this period. This can be motivated by the practical consideration that the destination terminal may be engaged in data transmission to another terminal during this period [12]. ${ }^{1}$ In the second signaling interval, both the relay and source terminals communicate with the destination terminal. For the $R \rightarrow D$ link, AF relaying is used, in which the relay terminal amplifies and retransmits the signal received from the source terminal in the first signaling interval. All terminals are equipped with a single transmit and receive antenna. Any linear modulation technique such as QAM or PSK modulation can be used.

Information symbols are first parsed to two streams of $M \times 1$ blocks $\mathbf{x}_{i}^{j}, i=1,2$ and then multiplied by a zero-padding (ZP) matrix $^{2} \Psi=\left[\mathbf{I}_{M}^{\mathrm{T}}, \mathbf{0}_{M \times \ell}^{\mathrm{T}}\right]^{\mathrm{T}}$ of size $N \times M$, where $\ell=\max \left(L_{1}+L_{3}, L_{2}\right)$ and $N$ is the frame length. As explained in [23], the use of zero-padding as the precoding method in a single-carrier transmission scenario ensures that the available multipath diversity is fully exploited. To further remove interblock interference and make the channel matrix circulant, a cyclic prefix (CP) with length $\ell$ is added between adjacent information blocks. Due to the adopted precoding form, i.e., zero padding, we simply insert additional zeros at the start of the frame as CP. In practical implementation, the block of all-zeros at the end of the current frame can also be used as the next frame's block of all-zeros to be inserted at its beginning, avoiding unnecessary additional overhead.

The signal received at the relay terminal during the first signaling interval is

$$
\mathbf{r}_{R}^{j}=\sqrt{E_{S R}} \mathbf{H}_{1}^{j} \boldsymbol{\Psi}_{1}^{j}+\mathbf{n}_{R}^{j}
$$

${ }^{1}$ It should be noted that the so-called Protocol I of [12] relaxes this assumption and considers source-to-destination transmission within the first signaling interval. A further modification of Protocol I is defined as Protocol II in [12] where only the relay terminal communicates with the destination terminal within the second signaling interval.

${ }^{2}$ The structure of the precoding matrix has the zero-padding form for D-TRSTBC and D-SC-STBC. For D-OFDM-STBC the precoding matrix is given by $\Psi=\mathbf{Q}^{\mathbf{H}}$, i.e., inverse Fourier transform (IFFT). where $\mathbf{H}_{1}^{j}$ is a $N \times N$ circulant matrix with entries $\left[\mathbf{H}_{1}^{j}\right]_{k, l}=$ $\mathbf{h}_{1}^{j}((k-l) \bmod N)$ and $\mathbf{n}_{R}^{j}$ is the additive white Gaussian noise vector with each entry having zero-mean and variance of $N_{0} / 2$ per dimension.

The relay terminal normalizes each entry of the received signal $\left[\mathbf{r}_{R}^{j}\right]_{n}, n=1,2, \ldots, N$ by a factor of $\mathrm{E}\left(\left|\left[\mathbf{r}_{R}^{j}\right]_{n}\right|^{2}\right)=E_{S R}+N_{0}$ to ensure unit average energy and retransmits the signal during the second time slot. Therefore, the received signal at the destination terminal in the second time slot is given by

$$
\mathbf{r}^{j}=\sqrt{E_{R D}} \mathbf{H}_{3}^{j} \tilde{\mathbf{r}}_{R}^{j}+\sqrt{E_{S D}} \mathbf{H}_{2}^{j} \mathbf{\Psi} \mathbf{x}_{2}^{j}+\mathbf{n}_{D}^{j}
$$

where $\tilde{\mathbf{r}}_{R}^{j}$ is the normalized received signal and $\mathbf{H}_{2}^{j}, \mathbf{H}_{3}^{j}$ are $N \times$ $N$ circulant matrices with entries $\left[\mathbf{H}_{2}^{j}\right]_{k, l}=\mathbf{h}_{2}^{j}((k-l) \bmod N)$ and $\left[\mathbf{H}_{3}^{j}\right]_{k, l}=\mathbf{h}_{3}^{j}((k-l) \bmod N)$, respectively. In (1), (2), $\mathbf{n}_{R}^{j}$ and $\mathbf{n}_{D}^{j}$ are the additive white Gaussian noise vectors with each entry having zero-mean and variance of $N_{0} / 2$ per dimension. Finally, $E_{S R}, E_{S D}$, and $E_{R D}$ are the average energies available at the respective terminals which take into account possibly different path loss and shadowing effects between the $S \rightarrow R$, $S \rightarrow D$, and $R \rightarrow D$ links, respectively. Combining (1) and (2), we obtain

$$
\mathbf{r}^{j}=\sqrt{\frac{E_{R D} E_{S R}}{E_{S R}+N_{0}}} \mathbf{H}_{3}^{j} \mathbf{H}_{1}^{j} \Psi \mathbf{x}_{1}^{j}+\sqrt{E_{S D}} \mathbf{H}_{2}^{j} \Psi \mathbf{x}_{2}^{j}+\tilde{\mathbf{n}}^{j}
$$

where we define the effective noise term as

$$
\tilde{\mathbf{n}}_{j}=\sqrt{\frac{E_{R D}}{E_{S R}+N_{0}}} \mathbf{H}_{3}^{j} \mathbf{n}_{R}^{j}+\mathbf{n}_{D}^{j}
$$

Each entry of effective noise term $\tilde{\mathbf{n}}_{j}$ (conditioned on $\mathbf{h}_{3}$ ) has zero mean and a variance of

$$
E\left[\left|\tilde{\mathbf{n}}^{j}\right|_{n}^{2} \mid \mathbf{h}_{3}^{j}\right]=N_{0}\left(1+\frac{E_{R D}}{E_{S R}+N_{0}} \sum_{m=0}^{L_{3}}\left|\mathbf{h}_{3}^{j}(m)\right|^{2}\right) .
$$

The destination terminal normalizes the received signal by a factor of $\sqrt{1+\sum_{m=0}^{L_{3}}\left|\mathbf{h}_{3}^{j}(m)\right|^{2} E_{R D} /\left(E_{S R}+N_{0}\right)}$. This does not affect the signal-to-noise ratio (SNR), but simplifies the ensuing presentation [12]. After normalization, we have

$$
\mathbf{r}^{j}=\sqrt{\gamma_{1}} \mathbf{H}_{3}^{j} \mathbf{H}_{1}^{j} \boldsymbol{\Psi} \mathbf{x}_{1}^{j}+\sqrt{\gamma_{2}} \mathbf{H}_{2}^{j} \Psi \mathbf{x}_{2}^{j}+\mathbf{n}^{j}
$$

where $\mathbf{n}^{j}$ is complex Gaussian with zero mean and variance of $N_{0} / 2$ per dimension. In (6), $\gamma_{1}$ and $\gamma_{2}$ are defined as

$$
\begin{aligned}
\gamma_{1}^{j} & =\frac{\left(E_{S R} / N_{0}\right) E_{R D}}{1+E_{S R} / N_{0}+\sum_{m=0}^{L_{3}}\left|\mathbf{h}_{3}^{j}(m)\right|^{2} E_{R D} / N_{0}} \\
\gamma_{2}^{j} & =\frac{\left(1+E_{S R} / N_{0}\right) E_{S D}}{1+E_{S R} / N_{0}+\sum_{m=0}^{L_{3}}\left|\mathbf{h}_{3}^{j}(m)\right|^{2} E_{R D} / N_{0}} .
\end{aligned}
$$

\section{D-TR STBC}

TR-STBC was introduced in [1] as an extension of the Alamouti STBC scheme [7] to frequency-selective channels by imposing the Alamouti orthogonal structure at a block level 


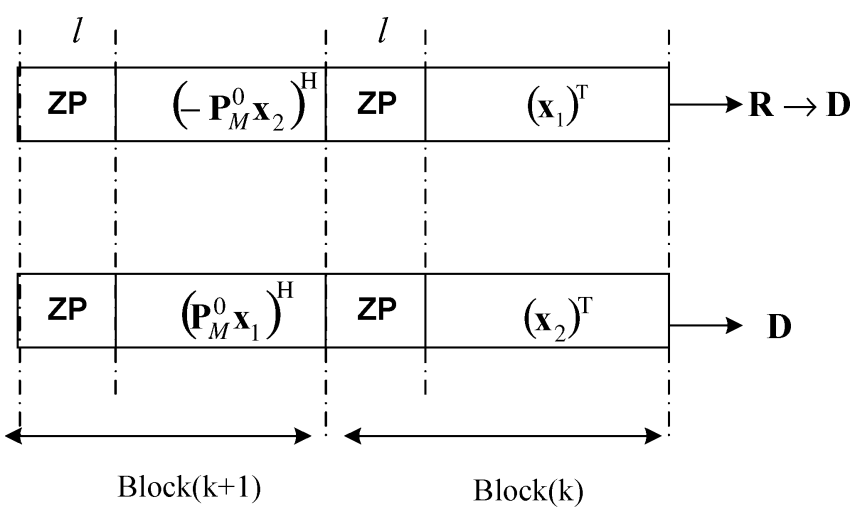

Fig. 2. Transmission block format for D-TR-STBC and D-SC-STBC.

rather than the original symbol-level implementation used for frequency-flat fading channels. We consider TR-STBC in a distributed fashion where the transmitted blocks for $S \rightarrow R$ and $S \rightarrow D$ links are generated by the encoding rule (cf. Fig. 2)

$$
\mathbf{d}_{1}^{k+1}=-\mathbf{J} \overline{\mathbf{d}}_{2}^{k}, \quad \mathbf{d}_{2}^{k+1}=\mathbf{J} \overline{\mathbf{d}}_{1}^{k}, \quad k=0,2,4, \ldots
$$

where $\mathbf{d}_{i}=\mathbf{\Psi}_{\mathbf{x}_{i}}, i=1,2$ is the zero-padded information vector and $\mathbf{J}=\mathbf{P}_{N}^{M}$ is a $N \times N$ partial permutation matrix. Assuming that the channel coefficients remain constant over blocks $k$ and $k+1$, i.e., $\mathbf{H}_{i}^{k}=\mathbf{H}_{i}^{k+1}=\mathbf{H}_{i}, i=1,2,3$, the received signals are given by

$$
\begin{aligned}
& \mathbf{r}^{k}=\sqrt{\gamma_{1}} \mathbf{H}_{3} \mathbf{H}_{1} \mathbf{d}_{1}^{k}+\sqrt{\gamma_{2}} \mathbf{H}_{2} \mathbf{d}_{2}^{k}+\mathbf{n}^{k} \\
& \mathbf{r}^{k+1}=-\sqrt{\gamma_{1}} \mathbf{H}_{3} \mathbf{H}_{1} \mathbf{J} \overline{\mathbf{d}}_{2}^{k}+\sqrt{\gamma_{2}} \mathbf{H}_{2} \mathbf{J} \overline{\mathbf{d}}_{1}^{k}+\mathbf{n}^{k+1} \text {. }
\end{aligned}
$$

Taking the conjugate of $\mathbf{r}^{k+1}$ and then multiplying by the permutation matrix $\mathbf{J}$, we have

$$
\mathbf{J} \overline{\mathbf{r}}^{k+1}=-\sqrt{\gamma_{1}} \mathbf{H}_{3}^{\mathrm{H}} \mathbf{H}_{1}^{\mathrm{H}} \mathbf{d}_{2}^{k}+\sqrt{\gamma_{2}} \mathbf{H}_{2}^{\mathrm{H}} \mathbf{d}_{1}^{k}+\mathbf{J} \overline{\mathbf{n}}^{k+1}
$$

where we use the identities $\mathbf{J} \overline{\mathbf{H}}_{3} \overline{\mathbf{H}}_{1} \mathbf{J}=\mathbf{H}_{3}^{\mathrm{H}} \mathbf{H}_{1}^{\mathrm{H}}$ and $\mathbf{J} \overline{\mathbf{H}}_{2} \mathbf{J}=$ $\mathbf{H}_{2}^{\mathrm{H}}$. Combining (10) and (12) in matrix form yields

$$
\left[\begin{array}{c}
\mathbf{r}^{k} \\
\tilde{\mathbf{r}}^{k+1}
\end{array}\right]=\underbrace{\left[\begin{array}{cc}
\sqrt{\gamma_{1} \mathbf{H}_{3} \mathbf{H}_{1}} & \sqrt{\gamma_{2} \mathbf{H}_{2}} \\
\sqrt{\gamma_{2}} \mathbf{H}_{2}^{\mathrm{H}} & -\sqrt{\gamma_{1} \mathbf{H}_{3}^{\mathrm{H}} \mathbf{H}_{1}^{\mathrm{H}}}
\end{array}\right]}_{\mathbf{H}_{e q}}\left[\begin{array}{c}
\mathbf{d}_{1}^{k} \\
\mathbf{d}_{2}^{k}
\end{array}\right]+\left[\begin{array}{c}
\mathbf{n}^{k} \\
\mathbf{J} \overline{\mathbf{n}}^{k+1}
\end{array}\right]
$$

where $\tilde{\mathbf{r}}^{k+1}=\mathbf{J} \overline{\mathbf{r}}^{k+1}$. Multiplying (13) by $\boldsymbol{\Upsilon}=\left(\mathbf{I}_{2} \otimes\right.$ $\left.\left(\sqrt{\gamma_{1}\left|\mathbf{H}_{3}\right|^{2}\left|\mathbf{H}_{1}\right|^{2}+\gamma_{2}\left|\mathbf{H}_{2}\right|^{2}}\right)^{-1}\right) \mathbf{H}_{e q}^{\mathrm{H}}$, we observe that the output streams are decoupled (due to orthogonality of $\mathbf{H}_{e q}$ ) allowing us to write

$$
\mathbf{r}_{\text {out }, i}^{k}=\sqrt{\gamma_{1}\left|\mathbf{H}_{3}\right|^{2}\left|\mathbf{H}_{1}\right|^{2}+\gamma_{2}\left|\mathbf{H}_{2}\right|^{2}} \mathbf{d}_{i}^{k}+\mathbf{n}_{\text {out }, i}^{k}, \quad i=1,2
$$

where $\mathbf{n}_{\text {out }, i}^{k}$ represents the filtered noise vector which is still Gaussian with each entry having zero-mean and a variance of $N_{0} / 2$ per dimension. Since the two data streams $\mathbf{d}_{1}^{k}$ and $\mathbf{d}_{2}^{k}$ are now decoupled, each can be detected by applying standard equalization techniques such as minimum mean square error (MMSE) or maximum likelihood sequence estimation (MLSE) equalizers [24].

\section{A. Diversity Gain Analysis}

Here, we investigate the achievable diversity gain for the D-TR-STBC scheme through the derivation of the PEP expression. PEP is the building block for the derivation of union bounds to the error probability. It is widely used in the literature to predict the attainable diversity order where the closed-form error probability expressions are unavailable.

First, we assume that $S \rightarrow R$ and $S \rightarrow D$ links experience frequency-selectivity while the channel between the relay and the destination terminals is AWGN, i.e., $\mathbf{h}_{3}^{j}[0]=1$. Physically, this assumption corresponds to the case where the destination and relay terminals have a very strong line-of-sight connection [12]. We should emphasize that this rather unrealistic assumption of static (i.e., nonfading) $R \rightarrow D$ link is made only to simplify performance analysis since it is difficult to analyze the general case where all underlying links experience frequency-selective fading. However, as we will demonstrate later, under certain assumptions imposed on the SNR of the underlying links, the PEP derivation for the general case becomes also analytically tractable.

Defining the transmitted codeword vector and the erroneously-decoded codeword vector as $\mathbf{x}$ and $\hat{\mathbf{x}}$, respectively, the conditional PEP is given as

$$
P\left(\mathbf{x}, \hat{\mathbf{x}} \mid \mathbf{h}_{1}^{j}, \mathbf{h}_{2}^{j}\right)=Q\left(\sqrt{\frac{d^{2}(\mathbf{x}, \hat{\mathbf{x}})}{2 N_{0}}}\right)
$$

assuming maximum likelihood (ML) decoding with perfect knowledge of the channel state information (CSI) at the receiver side. Here, $Q($.$) is the Gaussian- Q$ function and $d^{2}(\mathbf{x}, \hat{\mathbf{x}})$ denotes the Euclidean distance between $\mathbf{x}$ and $\hat{\mathbf{x}}$. Without loss of generality, we drop the the index $j$ in the PEP derivations for brevity. Applying the standard Chernoff bound to (15), we obtain

$$
P\left(\mathbf{x}, \hat{\mathbf{x}} \mid \mathbf{h}_{1}, \mathbf{h}_{2}\right) \leq \exp \left(-\frac{d^{2}(\mathbf{x}, \hat{\mathbf{x}})}{4 N_{0}}\right) .
$$

Noting that $\mathbf{d}_{i}=\Psi_{i} \mathbf{x}_{i}$, we can write $d^{2}(\mathbf{x}, \hat{\mathbf{x}})$ as follows:

$$
d^{2}(\mathbf{x}, \hat{\mathbf{x}})=\gamma_{1}\left\|\mathbf{H}_{1}(\mathbf{d}-\hat{\mathbf{d}})\right\|^{2}+\gamma_{2}\left\|\mathbf{H}_{2}(\mathbf{d}-\hat{\mathbf{d}})\right\|^{2}
$$

where $\gamma_{1}$ and $\gamma_{2}$ in (7) and (8) now reduce to

$$
\begin{aligned}
\gamma_{1} & =\frac{\left(E_{S R} / N_{0}\right) E_{R D}}{1+E_{S R} / N_{0}+E_{R D} / N_{0}} \\
\gamma_{2} & =\frac{\left(1+E_{S R} / N_{0}\right) E_{S D}}{1+E_{S R} / N_{0}+E_{R D} / N_{0}}
\end{aligned}
$$

under the static $R \rightarrow D$ link assumption and further defining

$$
\chi_{i}=\left[\begin{array}{cccc}
{[\mathbf{d}]_{0}} & {[\mathbf{d}]_{1}} & \cdots & {[\mathbf{d}]_{N-1}} \\
{[\mathbf{d}]_{N-1}} & {[\mathbf{d}]_{0}} & \cdots & {[\mathbf{d}]_{N-2}} \\
\vdots & \vdots & \cdots & \vdots \\
{[\mathbf{d}]_{N-L_{i}}} & {[\mathbf{d}]_{N-L_{i}+1}} & \cdots & {[\mathbf{d}]_{N-L_{i}-1}}
\end{array}\right]
$$


for $i=1,2,(17)$ can be rewritten as

$$
d^{2}(\mathbf{x}, \hat{\mathbf{x}})=\gamma_{1}\left\|\mathbf{h}_{1}^{\mathrm{T}}\left(\boldsymbol{\chi}_{1}-\hat{\boldsymbol{\chi}}_{1}\right)\right\|^{2}+\gamma_{2}\left\|\mathbf{h}_{2}^{\mathrm{T}}\left(\boldsymbol{\chi}_{2}-\hat{\boldsymbol{\chi}}_{2}\right)\right\|^{2} .
$$

Substituting (21) in (16) and following the steps detailed in Appendix I, we obtain the final PEP form as

$$
\begin{aligned}
P(\mathbf{x}, \hat{\mathbf{x}}) \leq\left(1+\frac{\gamma_{1}}{4 N_{0}}\right)^{-\left(L_{1}+1\right)} & \left(1+\frac{\gamma_{2}}{4 N_{0}}\right)^{-\left(L_{2}+1\right)} \\
\times & \prod_{l_{1}=0}^{L_{1}} \frac{1}{\boldsymbol{\lambda}_{1}\left(l_{1}\right)} \prod_{l_{2}=0}^{L_{2}} \frac{1}{\lambda_{2}\left(l_{2}\right)}
\end{aligned}
$$

where $\lambda_{i}, i=1,2$ denote the eigenvalues of the codeword difference matrix defined by $\mathrm{A}_{i}=\boldsymbol{\Omega}_{i}^{1 / 2} \boldsymbol{\chi}_{i} \boldsymbol{\Omega}_{i}^{1 / 2}$ with $\boldsymbol{\Omega}_{i}=$ $\operatorname{diag}\left(\mathbf{v}_{i}\right)$ and $\boldsymbol{\chi}_{i}=\left(\boldsymbol{\chi}_{i}-\hat{\boldsymbol{\chi}}_{i}\right)\left(\boldsymbol{\chi}_{i}-\hat{\boldsymbol{\chi}}_{i}\right)^{\mathrm{H}}$.

Maximum Achievable Diversity for D-TR-STBC: We assume perfect power control where $S \rightarrow D$ and $R \rightarrow D$ links are balanced and high SNRs for all underlying links, i.e., $E_{S D} / N_{0}=$ $E_{R D} / N_{0} \gg 1$. It is also assumed that SNR in $S \rightarrow R$ is large enough, i.e., $E_{S R} / N_{0}>E_{S D} / N_{0}$. Under these assumptions, we have $\gamma_{1} / N_{0}=\gamma_{2} / N_{0} \gg 1$, simplifying (22) to

$P(\mathbf{x}, \hat{\mathbf{x}}) \leq\left(\frac{E_{S D}}{4 N_{0}}\right)^{-\left(L_{1}+L_{2}+2\right)} \prod_{l_{1}=0}^{L_{1}} \frac{1}{\lambda_{1}\left(l_{1}\right)} \prod_{l_{2}=0}^{L_{2}} \frac{1}{\lambda_{2}\left(l_{2}\right)}$.

Since $\mathbf{A}_{i}$ is full rank, the maximum achievable diversity order is given by $L_{1}+L_{2}+2$. Under the assumption of nonfading $S \rightarrow R$ link, the maximum achievable diversity order can be similarly found and is given by $L_{3}+L_{2}+2$. If we further assume that $L_{1}=L_{2}=L$ in (23) (or similarly $L_{2}=L_{3}=L$ for the scenario with nonfading $S \rightarrow R$ link), we obtain

$$
P(\mathbf{x}, \hat{\mathbf{x}}) \leq\left(\frac{E_{S D}}{4 N_{0}}\right)^{-2(L+1)} \prod_{l=0}^{L}\left(\frac{1}{\lambda_{1}(l)}\right)^{2}
$$

which is the PEP expression for nondistributed TR-STBC.

Existence of Error Floor: In the investigation of the maximum achievable diversity order, we have assumed that SNR in $S \rightarrow R$ link is sufficiently large, i.e., $E_{S R} / N_{0}>E_{S D} / N_{0}$. Now we consider the limiting case of $E_{S D} / N_{0} \rightarrow \infty$. For this case, (22) takes the following form:

$P(\mathbf{x}, \hat{\mathbf{x}}) \leq\left(\frac{E_{S R}}{4 N_{0}}\right)^{-\left(L_{1}+L_{2}+2\right)} \prod_{l_{1}=0}^{L_{1}} \frac{1}{\lambda_{1}\left(l_{1}\right)} \prod_{l_{2}=0}^{L_{2}} \frac{1}{\lambda_{2}\left(l_{2}\right)}$.

It is observed that the performance becomes independent of $E_{S D} / N_{0}$ and is now governed by $E_{S R} / N_{0}$. Therefore, the performance is expected to deteriorate for low $E_{S R} / N_{0}$ resulting in error floors, which is an inherent disadvantage of the employed user cooperation protocol of [12], [13]. It should be noted that such error floors can be avoided if Protocol I of [12] is used as the user cooperation protocol where the source-to-destination transmission is allowed within the first signaling interval.
Effect of Power Imbalance: Now we consider the case of no power control and assume $E_{S D}=n E_{R D}$ where $n$ is a positive number. Under this assumption, PEP takes the form of

$$
\begin{aligned}
P(\mathbf{x}, \hat{\mathbf{x}}) \leq & \left(1+\frac{\frac{1}{n} \frac{E_{S R}}{N_{0}} \frac{E_{S D}}{4 N_{0}}}{1+\frac{E_{S R}}{N_{0}}+\frac{1}{n} \frac{E_{S D}}{N_{0}}}\right)^{-\left(L_{1}+1\right)} \\
& \times\left(1+\frac{\left(1+\frac{E_{S R}}{N_{0}}\right) \frac{E_{S D}}{4 N_{0}}}{1+\frac{E_{S R}}{N_{0}}+\frac{1}{n} \frac{E_{S D}}{N_{0}}}\right)^{-\left(L_{2}+1\right)} \\
& \times \prod_{l_{1}=0}^{L_{1}} \frac{1}{\lambda_{1}\left(l_{1}\right)} \prod_{l_{2}=0}^{L_{2}} \frac{1}{\lambda_{2}\left(l_{2}\right) .}
\end{aligned}
$$

For large $n,(26)$ reduces to

$$
\begin{aligned}
P(\mathbf{x}, \hat{\mathbf{x}}) \leq\left(1+\frac{1}{n} \frac{E_{S D}}{4 N_{0}}\right)^{-\left(L_{1}+1\right)} & \left(1+\frac{E_{S D}}{4 N_{0}}\right)^{-\left(L_{2}+1\right)} \\
& \times \prod_{l_{1}=0}^{L_{1}} \frac{1}{\lambda_{1}\left(l_{1}\right)} \prod_{l_{2}=0}^{L_{2}} \frac{1}{\lambda_{2}\left(l_{2}\right)}
\end{aligned}
$$

where the performance is governed by $E_{S D} / N_{0}$ which means that the $S \rightarrow D$ link is the dominant link. On the other hand, for small values of $n,(26)$ reduces to

$$
\begin{aligned}
P(\mathbf{x}, \hat{\mathbf{x}}) \leq\left(1+\frac{E_{S R}}{4 N_{0}}\right)^{-\left(L_{1}+1\right)} & \left(1+\frac{n}{4}\left(1+\frac{E_{S R}}{N_{0}}\right)\right)^{-\left(L_{2}+1\right)} \\
& \times \prod_{l_{1}=0}^{L_{1}} \frac{1}{\lambda_{1}\left(l_{1}\right)} \prod_{l_{2}=0}^{L_{2}} \frac{1}{\lambda_{2}\left(l_{2}\right)}
\end{aligned}
$$

where the performance is once again dominated by $E_{S R} / N_{0}$.

Next, we focus on the general case where all three underlying links experience frequency-selective fading. Due to the presence of $\left|\mathbf{h}_{3}(m)\right|^{2}$ terms in (7) and (8), the derivation of PEP becomes analytically intractable without any assumptions imposed on the SNR in the underlying links. However, for the asymptotic case of $E_{S D} / N_{0}=E_{R D} / N_{0} \gg 1$ with perfect power control and sufficiently large $E_{S R} / N_{0}>E_{S D} / N_{0}$ values, the scaling factors in (7) and (8) reduce to $\gamma_{1}=\gamma_{2}=E_{S D}$. Then, we can obtain the following PEP expressions (see Appendix II for detailed steps of the derivations):

Case 1) $L_{3}>L_{1}$

$$
\begin{aligned}
P(\mathbf{x}, \hat{\mathbf{x}}) \leq\left(\frac{E_{S D}}{4 N_{0}}\right)^{-\left(L_{1}+L_{2}+2\right)} & \left(L_{3}+1\right)^{L_{1}} \frac{\Gamma\left(L_{3}-L_{1}+1\right)}{\Gamma\left(L_{3}+1\right)} \\
& \times \prod_{l_{1}=0}^{L_{1}}\left(\boldsymbol{\lambda}_{1}\left(l_{1}\right)\right)^{-1} \prod_{l_{2}=0}^{L_{2}}\left(\boldsymbol{\lambda}_{2}\left(l_{2}\right)\right)^{-1}
\end{aligned}
$$

Case 2) $L_{1}>L_{3}$

$$
\begin{aligned}
P(\mathbf{x}, \hat{\mathbf{x}}) \leq\left(\frac{E_{S D}}{4 N_{0}}\right)^{-\left(L_{3}+L_{2}+2\right)}\left(L_{1}+1\right)^{L_{3}} \frac{\Gamma\left(L_{1}-L_{3}+1\right)}{\Gamma\left(L_{1}+1\right)} \\
\times \prod_{l_{3}=0}^{L_{3}}\left(\boldsymbol{\lambda}_{1}\left(l_{3}\right)\right)^{-1} \prod_{l_{2}=0}^{L_{2}}\left(\boldsymbol{\lambda}_{2}\left(l_{2}\right)\right)^{-1}
\end{aligned}
$$


TABLE I

ACHIEVABLE DIVERSITY ORDERS FOR DISTRIBUTED TR-STBC AND SC-STBC

$\begin{array}{llll}\mathrm{S} \rightarrow R & \mathrm{R} \rightarrow D & \mathrm{~S} \rightarrow D & \\ \text { Fading } & \text { Fading } & \text { Fading } & \min \left(L_{1}, L_{3}\right)+L_{2}+2 \\ \text { Fading } & \text { Non-fading } & \text { Fading } & L_{1}+L_{2}+2 \\ \text { Non-fading } & \text { Fading } & \text { Fading } & L_{3}+L_{2}+2\end{array}$

Case 3) $L_{1}=L_{3}$

$$
\begin{aligned}
P(\mathbf{x}, \hat{\mathbf{x}}) \leq\left(\frac{E_{S D}}{4 N_{0}}\right)^{-\left(L_{3}+L_{2}+2\right)} & \frac{\left(L_{3}+1\right)^{L_{3}+1}}{\Gamma\left(L_{3}+1\right)}(-1)^{L_{3}-1} \\
& \times \prod_{l_{2}=0}^{L_{2}}\left(\boldsymbol{\lambda}_{2}\left(l_{2}\right)\right)^{-1} \sum_{l_{1}=1}^{L_{1}+1} \frac{p_{l_{1}}}{\left(\lambda_{1}\left(l_{1}\right)\right)^{L_{3}+1}} e^{\beta} E_{i}(-\beta) .
\end{aligned}
$$

In (29)-(31), $\Gamma[$.$] and E_{i}[$.$] represent the gamma function and$ the exponential-integral function, respectively, [25]. It is observed from (29)-(31) that the maximum achievable diversity order is given by $\min \left(L_{1}, L_{3}\right)+L_{2}+2$. This illustrates that the smaller of the multipath diversity orders experienced in $S \rightarrow R$ and $R \rightarrow D$ links becomes the performance bottleneck for the relaying path. In other words, it is not possible to extract the full multipath diversity for D-TR-STBC in a cooperative scenario. A summery of our results on the achievable diversity orders is provided in Table I. The term "fading" in the table is used to refer to both frequency-flat and frequency-selective channels since the derived expressions in (29)-(31) cover frequency-flat fading as a special case.

\section{D-SC STBC}

Single-carrier frequency domain equalization is an attractive equalization scheme for broadband wireless channels which are characterized by their long impulse response memory [26]. An Alamouti-based scheme for frequency-selective channels which relies on frequency-domain equalization was proposed in [2]. Here, we consider its distributed version within the context of our relay-assisted transmission scenario (cf. Fig. 2). Assuming that the channel coefficients remain constant over two consecutive blocks, i.e. $\mathbf{H}_{i}^{k}=\mathbf{H}_{i}^{k+1}=\mathbf{H}_{i}$ for $i=1,2,3$, the received signals for blocks $k$ and $k+1$ are

$$
\begin{aligned}
& \mathbf{r}^{k}=\sqrt{\gamma_{1}} \mathbf{H}_{3} \mathbf{H}_{1} \mathbf{d}_{1}^{k}+\sqrt{\gamma_{2}} \mathbf{H}_{2} \mathbf{d}_{2}^{k}+\mathbf{n}^{k} \\
& \mathbf{r}^{k+1}=-\sqrt{\gamma_{1}} \mathbf{H}_{3} \mathbf{H}_{1} \mathbf{J} \overline{\mathbf{d}}_{2}^{k}+\sqrt{\gamma_{2}} \mathbf{H}_{2} \mathbf{J} \overline{\mathbf{d}}_{1}^{k+1}+\mathbf{n}^{k+1}
\end{aligned}
$$

which are identical to (10) and (11) and repeated here for convenience. Next, we transform the received signals to the frequency domain by applying the Discrete Fourier Transform (DFT), ${ }^{3}$ i.e., multiplying by the $\mathbf{Q}$ matrix

$$
\begin{aligned}
\mathbf{Q r}^{k}= & \sqrt{\gamma_{1}} \mathbf{Q} \mathbf{H}_{3} \mathbf{H}_{1} \mathbf{d}_{1}^{k}+\sqrt{\gamma_{2}} \mathbf{Q} \mathbf{H}_{2} \mathbf{d}_{2}^{k}+\mathbf{Q n}^{k} \\
\mathbf{Q J}^{k+1}= & -\sqrt{\gamma_{1}} \mathbf{Q} \mathbf{H}_{3}^{\mathrm{H}} \mathbf{H}_{1}^{\mathrm{H}} \mathbf{d}_{2}^{k} \\
& +\sqrt{\gamma_{2}} \mathbf{Q} \mathbf{H}_{2}^{\mathrm{H}} \mathbf{d}_{1}^{k}+\mathbf{Q} \overline{\mathbf{J}}^{k+1} .
\end{aligned}
$$

${ }^{3}$ In this paper, we assume that the DFT size $N$ is a power of 2, thus, the terms FFT and DFT are interchangeable.
Exploiting the circulant structure of the channel matrices, we have

$$
\mathbf{H}_{i}^{j}=\mathbf{Q}^{\mathrm{H}} \mathbf{\Lambda}_{i}^{j} \mathbf{Q}
$$

where $\Lambda_{i}^{j}, i=1,2,3$, is a diagonal matrix whose $(n, n)$ element is equal to the $n^{\text {th }}$ DFT coefficient of $\mathbf{h}_{i}^{j}$. Using (36), we can write (34) and (35) in matrix form a

$$
\begin{array}{r}
{\left[\begin{array}{c}
\mathbf{Q r}^{k} \\
\mathbf{Q J} \overline{\mathbf{r}}^{k+1}
\end{array}\right]=\underbrace{\left[\begin{array}{cc}
\sqrt{\gamma_{1}} \boldsymbol{\Lambda}_{3} \boldsymbol{\Lambda}_{1} & \sqrt{\gamma_{2}} \boldsymbol{\Lambda}_{2} \\
\sqrt{\gamma_{2}} \overline{\boldsymbol{\Lambda}}_{2} & -\sqrt{\gamma_{1}} \overline{\boldsymbol{\Lambda}}_{3} \overline{\boldsymbol{\Lambda}}_{1}
\end{array}\right]}_{\tilde{\boldsymbol{\Lambda}}}\left[\begin{array}{c}
\mathbf{Q} \mathbf{d}_{1}^{k} \\
\mathbf{Q d}_{2}^{k}
\end{array}\right]} \\
+\left[\begin{array}{c}
\mathbf{Q n}^{k} \\
\mathbf{Q J n}^{k+1}
\end{array}\right] .
\end{array}
$$

Since $\tilde{\Lambda}$ is an orthogonal matrix of size $2 N \times 2 N$, we can multiply (37) (without loss of optimality) by

$$
\mathbf{K}=\left(\mathbf{I}_{2} \otimes\left(\gamma_{1}\left|\boldsymbol{\Lambda}_{3}\right|^{2}\left|\boldsymbol{\Lambda}_{1}\right|^{2}+\gamma_{2}\left|\boldsymbol{\Lambda}_{2}\right|^{2}\right)^{-1 / 2}\right) \tilde{\Lambda}^{H} .
$$

The resulting output streams are now decoupled allowing us to write each output sequence as

$$
\mathbf{r}_{\text {out }, i}^{k}=\sqrt{\gamma_{1}\left|\boldsymbol{\Lambda}_{3}\right|^{2}\left|\boldsymbol{\Lambda}_{1}\right|^{2}+\gamma_{2}\left|\boldsymbol{\Lambda}_{2}\right|^{2}} \mathbf{Q} \mathbf{d}_{i}^{k}+\mathbf{n}_{\text {out }, i}^{k}
$$

where $\mathbf{n}_{\text {out }, i}^{k}$ is a noise vector with each entry still Gaussian with zero-mean and variance of $N_{0} / 2$ per dimension. After the decoupling, standard SISO equalizers can be used for the detection of data streams $\mathbf{x}_{i}^{k}, i=1,2$.

\section{A. Diversity Gain Analysis}

Here, we investigate the achievable diversity order for D-SCSTBC scheme through the derivation of the PEP expression. For a given channel realization, the Chernoff bound on PEP is given by (16) where $d^{2}(\mathbf{x}, \hat{\mathbf{x}})$ is

$$
d^{2}(\mathbf{x}, \hat{\mathbf{x}})=\|\mathbf{Y Q}(\mathbf{d}-\hat{\mathbf{d}})\|^{2}
$$

with $\mathbf{Y}=\sqrt{\gamma_{1}\left|\boldsymbol{\Lambda}_{1}\right|^{2}+\gamma_{2}\left|\boldsymbol{\Lambda}_{2}\right|^{2}}$. Then, (40) can be rewritten as

$$
\begin{aligned}
d^{2}(\mathbf{x}, \hat{\mathbf{x}}) & =\mathbf{e}^{\mathrm{H}} \mathbf{Q}^{\mathrm{H}} \mathbf{Y}^{\mathrm{H}} \mathbf{Y} \mathbf{Q e} \\
& =\gamma_{1} \mathbf{e}^{\mathrm{H}} \mathbf{Q}^{\mathrm{H}}\left|\boldsymbol{\Lambda}_{1}\right|^{2} \mathbf{Q} \mathbf{e}+\gamma_{2} \mathbf{e}^{\mathrm{H}} \mathbf{Q}^{\mathrm{H}}\left|\boldsymbol{\Lambda}_{2}\right|^{2} \mathbf{Q} \mathbf{e}
\end{aligned}
$$

where $\mathbf{e}=\mathbf{d}-\hat{\mathbf{d}}$. Using the property $\mathbf{Q}^{\mathrm{H}}\left|\boldsymbol{\Lambda}_{i}\right|^{2} \mathbf{Q}=\mathbf{H}_{i} \mathbf{H}_{i}^{\mathrm{H}}$, we write (41) as

$$
d^{2}(\mathbf{x}, \hat{\mathbf{x}})=\gamma_{1}\left\|\mathbf{H}_{1} \mathbf{e}\right\|^{2}+\gamma_{2}\left\|\mathbf{H}_{2} \mathbf{e}\right\|^{2}
$$

Interestingly, comparing (17) and (42), we notice that distance metrics for TR-STBC and SC-STBC turn out to be identical. Thus, following the same steps of the previous section, we obtain the same PEP expressions for D-TR-STBC, i.e., (22) and (29)-(31). Therefore, the achievable diversity orders for both schemes are the same (See Table I).

We should emphasize here that the equivalence between these two schemes (assuming maximum likelihood detection) has not been reported before in the literature (even for the noncooperative case) to the best of our knowledge. It is also interesting to note that, under the following assumptions 
- Nonfading $R \rightarrow D$ link;

- Perfect power control employed, i.e., $E_{S D}=E_{R D}$ and $E_{S R} / N_{0} \gg E_{S D} / N_{0}$

- High SNR, i.e., $\gamma_{1} / N_{0}=\gamma_{2} / N_{0}=E_{S D} / N_{0} \gg 1$;

- Equal channel memory lengths, i.e., $L=L_{1}=L_{2}$;

Equation (22) reduces to

$$
P(\mathbf{x}, \hat{\mathbf{x}}) \leq\left(\frac{E_{S D}}{4 N_{0}}\right)^{-2(L+1)} \prod_{l=0}^{L}\left(\frac{1}{\lambda_{1}(l)}\right)^{2}
$$

which was earlier reported in [27] for nondistributed SC-STBC.

\section{D-OFDM STBC}

In OFDM, the high-rate input stream is demultiplexed and transmitted over a number of low-rate independent frequency sub-carriers. This multicarrier transmission scheme can be efficiently implemented in practice using the fast Fourier transform (FFT). An elegant scheme for combining OFDM and STBC by implementing the Alamouti orthogonal structure at a block level was first reported in [3]. In this paper, we consider a similar transmission format as illustrated in Fig. 3. Since we assume one relay, the information data symbols are parsed to two streams of $N \times 1$ blocks $\mathbf{x}_{i}^{j} i=1,2$ and then precoded by $\Psi_{i}=\mathbf{Q}^{H}$ matrix where $\mathbf{Q}^{H}$ represents the inverse FFT (IFFT) matrix. The CP symbols here are the last $\tilde{L}=\max \left(L_{1}+L_{3}, L_{2}\right)$ symbols of the transmission blocks. The received signals can be written as

$$
\begin{aligned}
& \mathbf{r}^{k}=\sqrt{\gamma_{1}} \mathbf{H}_{3} \mathbf{H}_{1} \mathbf{Q}^{\mathrm{H}} \mathbf{x}_{1}^{k}+\sqrt{\gamma_{2}} \mathbf{H}_{2} \mathbf{Q}^{\mathrm{H}} \mathbf{x}_{2}^{k}+\mathbf{n}^{k}
\end{aligned}
$$

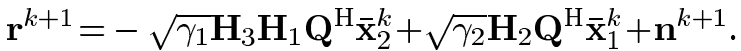

Applying the DFT operation to (44) and (45) and using (36), we have

$$
\begin{aligned}
\mathbf{Q r}^{k} & =\sqrt{\gamma_{1}} \boldsymbol{\Lambda}_{3} \boldsymbol{\Lambda}_{1} \mathbf{x}_{1}^{k}+\sqrt{\gamma_{2}} \boldsymbol{\Lambda}_{2} \mathbf{x}_{2}^{k}+\mathbf{Q n}^{k} \\
\mathbf{Q r}^{k+1} & =-\sqrt{\gamma_{1}} \boldsymbol{\Lambda}_{3} \boldsymbol{\Lambda}_{1} \overline{\mathbf{x}}_{2}^{k}+\sqrt{\gamma_{2}} \boldsymbol{\Lambda}_{2} \overline{\mathbf{x}}_{1}^{k}+\mathbf{Q} \mathbf{n}^{k+1}
\end{aligned}
$$

Combining (46) and the conjugated version of (47) in a matrix form, we obtain

$$
\begin{aligned}
{\left[\begin{array}{c}
\mathbf{Q r}^{k} \\
\mathbf{Q r}^{k+1}
\end{array}\right] } & =\left[\begin{array}{cc}
\sqrt{\gamma_{1}} \mathbf{\Lambda}_{\mathbf{3}} \mathbf{\Lambda}_{1} & \sqrt{\gamma_{2}} \mathbf{\Lambda}_{\mathbf{2}} \\
\sqrt{\gamma_{2}} \bar{\Lambda}_{\mathbf{2}} & -\sqrt{\gamma_{1}} \overline{\mathbf{\Lambda}}_{\mathbf{3}} \overline{\mathbf{\Lambda}}_{\mathbf{1}}
\end{array}\right]\left[\begin{array}{c}
\mathbf{x}_{1}^{k} \\
\mathbf{x}_{2}^{k}
\end{array}\right]+\left[\frac{\mathbf{Q n}^{k}}{\mathbf{Q n}^{k+1}}\right] \\
& =\tilde{\mathbf{\Lambda}} \mathbf{x}+\tilde{\mathbf{N}} .
\end{aligned}
$$

Multiplying (48) by (38) yields the decoupled streams

$$
\mathbf{r}_{\text {out }, i}^{k}=\sqrt{\gamma_{1}\left|\boldsymbol{\Lambda}_{3}\right|^{2}\left|\boldsymbol{\Lambda}_{1}\right|^{2}+\gamma_{2}\left|\boldsymbol{\Lambda}_{2}\right|^{2}} \mathbf{x}_{i}^{k}+\mathbf{n}_{\text {out }, i}^{k}, \quad i=1,2
$$

where $\mathbf{n}_{o u t, i}^{k}$ is complex Gaussian noise with zero-mean and variance of $N_{0} / 2$ per-dimension.

It is clear from the above descriptions that both OFDM and SC-FDE rely on FFT/IFFT operations. However, SC-FDE is distinct from OFDM in that the IFFT block is moved to the receiver end and placed before the decision device. This causes the effects of deep nulls in the channel frequency response (caused by the destructive addition of multipath) to spread out by the IFFT operation over all symbols, thus reducing their effect and improving the overall performance compared to uncoded OFDM.

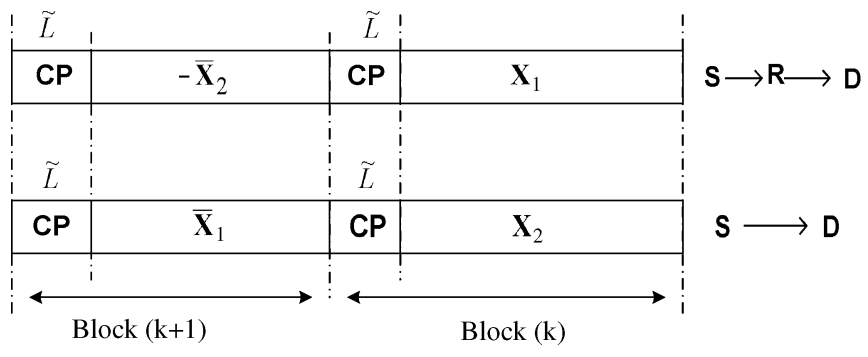

Fig. 3. Transmission block format for D-OFDM-STBC.

\section{A. Diversity Gain Analysis}

Here, we derive the PEP expression for the D-OFDM-STBC scheme. Under the following assumptions: $R \rightarrow D$ link is nonfading, i.e., $L_{3}=0$ and $\mathbf{h}_{3}[0]=1$, perfect power control where $S \rightarrow D$ and $R \rightarrow D$ links are balanced, high SNRs for all underlying links, i.e., $E_{S D} / N_{0}=E_{R D} / N_{0} \gg 1$ and $E_{S R} / N_{0}>$ $E_{S D} / N_{0}$, we follow the steps detailed in Appendix III and obtain the PEP for D-OFDM-STBC as

$$
P(\mathbf{x}, \hat{\mathbf{x}}) \leq\left(\frac{E_{S D}}{4 N_{0}}\right)^{-2} \frac{1}{\lambda_{\boldsymbol{\Sigma}_{1}}} \frac{1}{\lambda_{\boldsymbol{\Sigma}_{2}}}
$$

where $\lambda_{\boldsymbol{\Sigma}_{1}}, \lambda_{\boldsymbol{\Sigma}_{2}}$ are, respectively, the eigenvalues of $\boldsymbol{\Sigma}_{1}$ and $\boldsymbol{\Sigma}_{2}$ which are functions of the codeword difference matrices (See Appendix III). From (50), we conclude that uncoded D-OFDMSTBC achieves only a diversity order of two and fails to exploit the underlying multi-path diversity. For the general case where all underlying links experience frequency-selectivity and under similar assumptions on SNRs, we obtain the PEP expression as (See Appendix IV)

$$
P(\mathbf{x}, \hat{\mathbf{x}}) \leq\left(\frac{E_{S D}}{4 N_{0}}\right)^{-2} \frac{\Gamma\left(L_{3}\right)\left(L_{3}+1\right)}{\Gamma\left(L_{3}+1\right)} \frac{1}{\lambda_{\Sigma_{1}}} \frac{1}{\lambda_{\Sigma_{2}}} .
$$

It is seen from (51) that the diversity order of uncoded D-OFDM-STBC is again limited by two and is independent of the channel memory lengths $L_{1}, L_{2}$, and $L_{3}$. In other words, it extracts only the available spatial diversity, but fails to exploit the multipath diversity.

Outer coding with frequency interleaving can be combined with OFDM to extract the multipath diversity available in the considered cooperative scenario and, therefore, to further improve the performance. Although different outer codes can be employed, we consider trellis-coded modulation (TCM) [28] since it does not result in additional bandwidth expansion from the uncoded case. Previous works on the concatenation of TCM-STBC for the flat-fading channel case should also be noted [29]-[32].

For nonfading $R \rightarrow D$ link, the PEP for trellis coded D-OFDM-STBC is given by (see Appendix V)

$P(\mathbf{x}, \hat{\mathbf{x}}) \leq\left(\frac{E_{S D}}{4 N_{0}}\right)^{-\beta} \prod_{j_{1}=1}^{r_{1}}\left(\frac{1}{\lambda_{\Theta_{1}}\left(j_{1}\right)}\right)^{-1} \prod_{j_{2}=1}^{r_{2}}\left(\frac{1}{\lambda_{\Theta_{2}}\left(j_{2}\right)}\right)^{-1}$

Following a similar argument as in [33], it can be shown that the diversity order is

$$
\beta=\min \left(\lceil E C L / 2\rceil, L_{1}+1\right)+\min \left(\lceil E C L / 2\rceil, L_{2}+1\right)
$$


TABLE II

ACHIEVABLE DIVERSITY ORDERS FOR DISTRIBUTED OFDM-STBC

$\begin{array}{lllll}\mathrm{S} \rightarrow R & \mathrm{R} \rightarrow D & \mathrm{~S} \rightarrow D & \text { Uncoded } & \text { Coded } \\ \text { Fading } & \text { Fading } & \text { Fading } & 2 & \min \left(\lceil\mathrm{ECL} / 2\rceil, L_{1}+1, L_{3}+1\right)+\min \left(\lceil\mathrm{ECL} / 2\rceil, L_{2}+1\right) \\ \text { Fading } & \text { Non-fading } & \text { Fading } & 2 & \min \left(\lceil\mathrm{ECL} / 2\rceil, L_{1}+1\right)+\min \left(\lceil\mathrm{ECL} / 2\rceil, L_{2}+1\right) \\ \text { Non-fading } & \text { Fading } & \text { Fading } & 2 & \min \left(\lceil\mathrm{ECL} / 2\rceil, L_{3}+1\right)+\min \left(\lceil\mathrm{ECL} / 2\rceil, L_{2}+1\right)\end{array}$

where $E C L$ is the effective code length of the outer TCM code. For the general case where all links experience frequency-selectivity, it can be shown that the diversity order is

$\beta=\min \left(\lceil E C L / 2\rceil, L_{1}+1, L_{3}+1\right)+\min \left(\lceil E C L / 2\rceil, L_{2}+1\right)$

Our derivation points out that with an appropriate choice of underlying TCM code (i.e., with sufficiently large ECL), coded D-OFDM-STBC achieves the same diversity order as D-TRSTBC and D-SC-STBC. Therefore, using judiciously-designed outer codes with frequency-interleaving, both spatial and multipath diversity gains can be achieved in a coded D-OFDM-STBC scheme. ${ }^{4}$ A summary of our results on the achievable diversity orders for D-OFDM-STBC is provided in Table II.

\section{NUMERICAL RESULTS}

In this section, we present Monte Carlo simulation results for distributed STBC systems which have been described and analyzed in this paper. We assume a quasi-static Rayleigh fading channel and employ 4-PSK modulation.

First, we consider a scenario with a nonfading $R \rightarrow D$ link which can be justified in a practical scenario by the existence of a strong line-of-sight path. $S \rightarrow D$ and $S \rightarrow R$ links are modeled as frequency-selective channels with memory lengths $L_{1}=L_{2}=3$ and a uniform delay power profile. We further assume perfect power control, i.e., the $S \rightarrow D$ and $R \rightarrow D$ links are balanced. Under this scenario, Fig. 4 illustrates the symbol error rate (SER) performance of D-TR-STBC, D-SCSTBC, and uncoded D-OFDM-STBC for $E_{S R} / N_{0}=25 \mathrm{~dB}$. D-TR-STBC and D-SC-STBC yield identical performance and both schemes (with MLSE decoding) are able to provide a diversity order of $L_{1}+L_{2}+2=8$, confirming our conclusions from the PEP analysis, cf. (23). Although MLSE is able to collect the maximum achievable diversity gain, its complexity might be prohibitive for some cases. Therefore, we also consider MMSE in our simulations as an alternative suboptimum equalizer. Our simulation results indicate that the MLSE equalizer outperforms the MMSE equalizer by $\approx 2.7 \mathrm{~dB}$ at SER $=10^{-4}$. This loss can be further reduced, if multiple antennas are employed at the destination [27]. It is also observed from Fig. 4 that both schemes outperform uncoded D-OFDM-STBC significantly since the latter scheme is not able to exploit the underlying multipath diversity without channel coding and frequency interleaving as previously discussed. We notice from the figure that both D-TR-STBC and D-SC-STBC outperform D-OFDM-STBC by $\approx 4 \mathrm{~dB}$ and $6 \mathrm{~dB}$ at SER $=10^{-3}$ for MMSE and MLSE implementations, respectively.

\footnotetext{
${ }^{4}$ Besides the employment of outer coding, another alternative to extract multipath diversity is to use space-time-frequency (STF) codes proposed in [34] which cleverly exploit subgrouping technique to design low-complexity STF block codes. We do not pursue it here due to the space limitations.
}

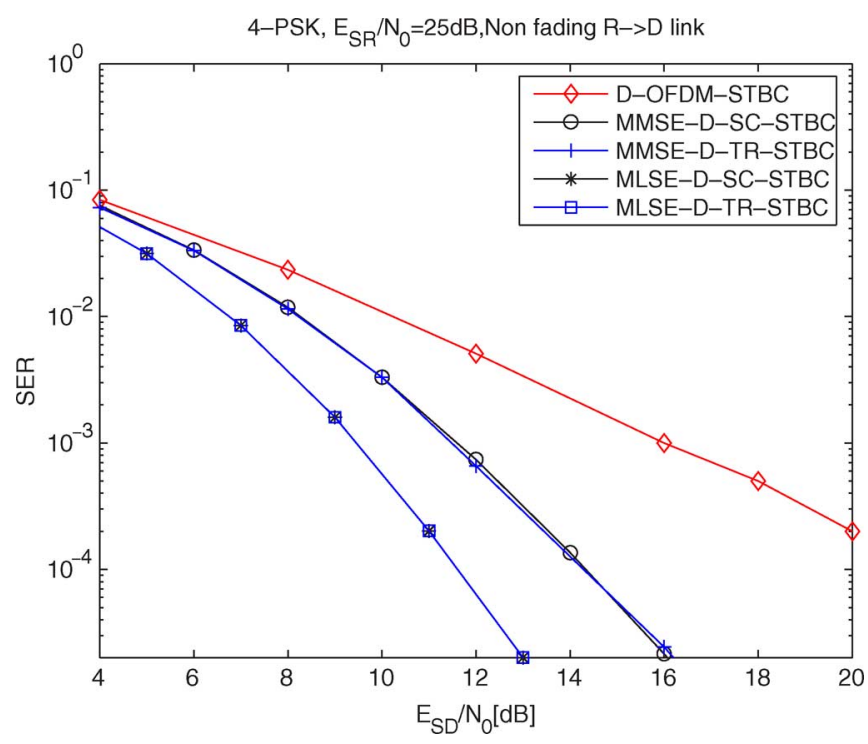

Fig. 4. SER performances of D-TR-STBC, D-SC-STBC, and D-OFDM STBC for nonfading $R \rightarrow D$ link $\left(E_{S R} / N_{0}=25 \mathrm{~dB}\right)$.

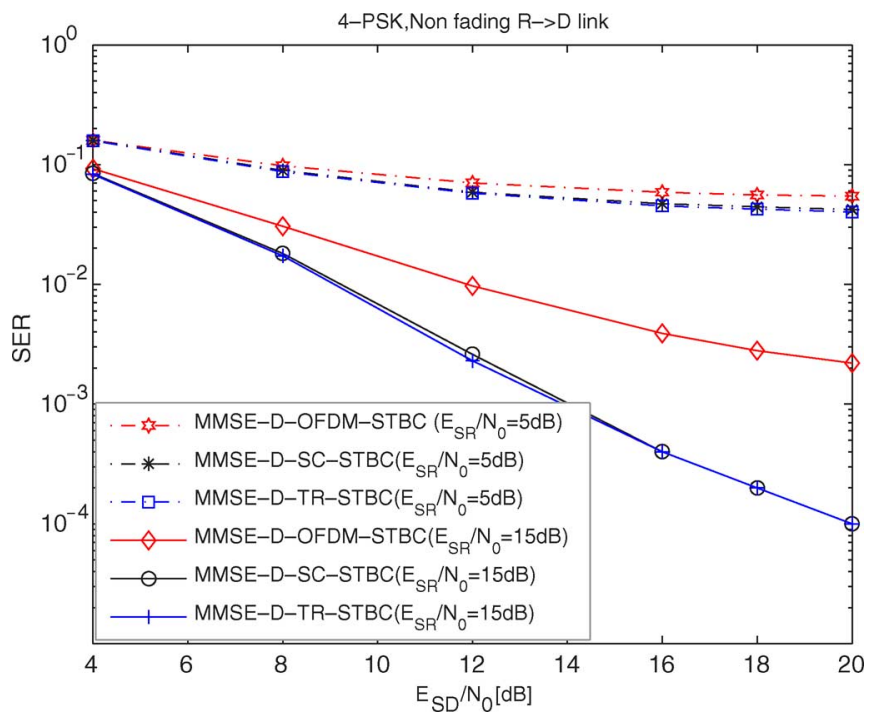

Fig. 5. SER performances of D-TR-STBC, D-SC-STBC, and D-OFDM STBC for nonfading $R \rightarrow D$ link. $\left(E_{S R} / N_{0}=5,15 \mathrm{~dB}\right)$.

Fig. 5 illustrates the performance of D-TR-STBC, D-SC-STBC, and uncoded D-OFDM-STBC for $E_{S R} / N_{0}=$ $5 \mathrm{~dB}$ and $E_{S R} / N_{0}=15 \mathrm{~dB}$. The performances of all three schemes are significantly degraded as $E_{S R} / N_{0}$ decreases, confirming our observations from (25). As noted previously, this is an inherent disadvantage of the adopted user cooperation protocol of [12], [13]. Relay-selection algorithms can be used to remove the error floor and to preserve the diversity order at an acceptable level [37]. 


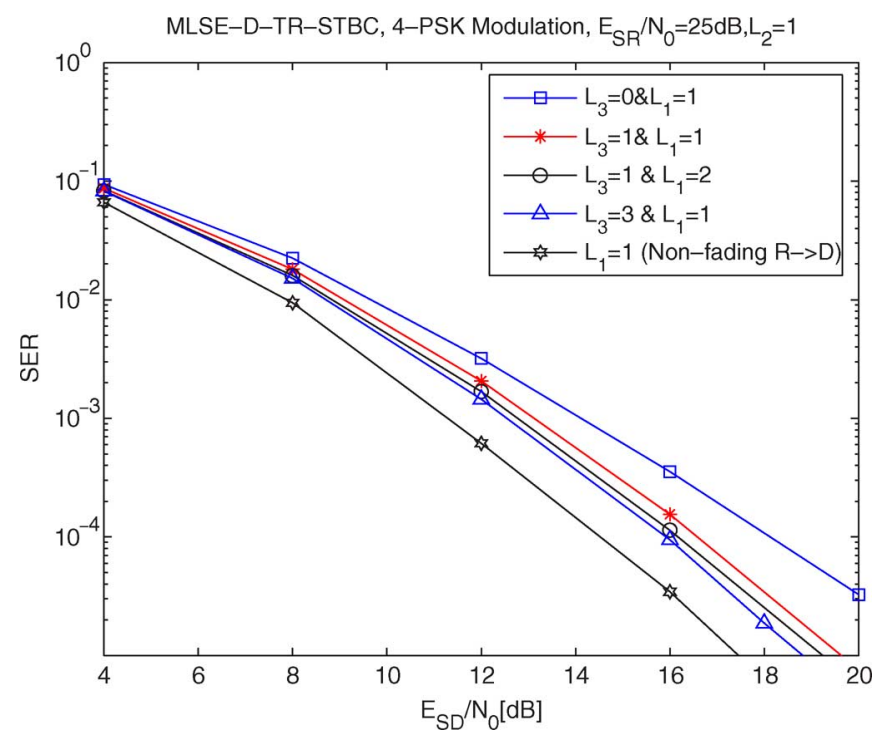

Fig. 6. SER performance of D-TR-STBC over frequency-selective $S \rightarrow R$, $R \rightarrow D$, and $S \rightarrow D$ links for various combinations of channel lengths.

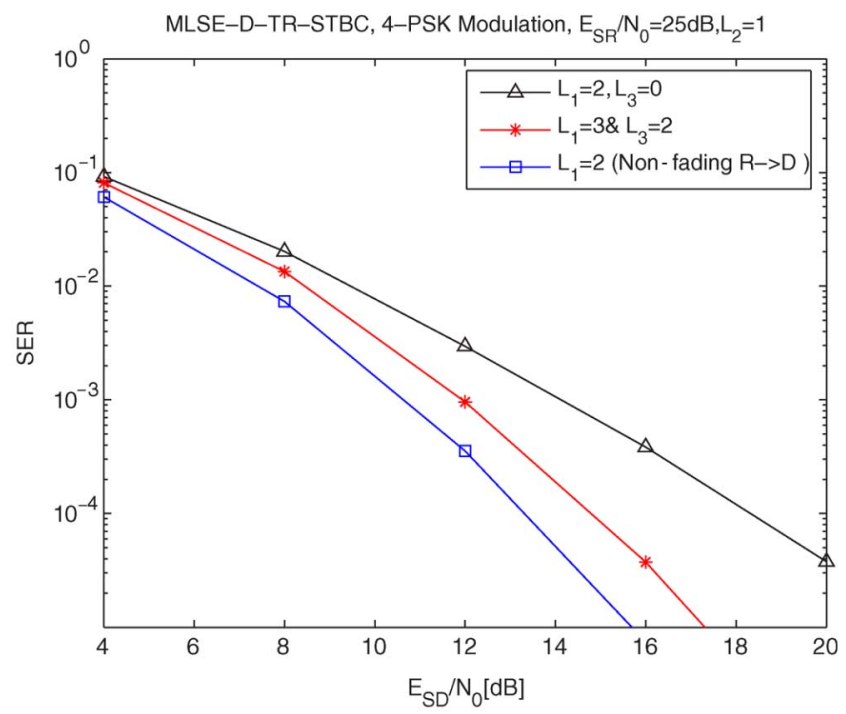

Fig. 7. SER performance of D-TR-STBC over frequency-selective $S \rightarrow R$, $R \rightarrow D$, and $S \rightarrow D$ links for various combinations of channel lengths.

In Figs. 6 and 7, we investigate the performance of D-TRSTBC assuming MLSE equalization and relaxing the assumption of nonfading $R \rightarrow D$ link. We assume various combinations of channel memory lengths. It should be noted here that interchanging the values of $L_{1}$ and $L_{3}$ yields identical performance and those symmetrical scenarios are omitted here for the sake of presentation simplicity. The case of nonfading $R \rightarrow D$ link, i.e., $L_{3}=0$ with $\mathbf{h}_{3}[0]=1$, is also included as a benchmark. It is clear from the slopes of the error rate performance curves in Figs. 6 and 7 that the maximum achievable diversity order is $\min \left(L_{1}, L_{3}\right)+L_{2}+2$ for each case, confirming our observations from the PEP expressions in (29)-(31). For example, in Fig. 6 for the considered scenarios of $\left(L_{1}=1\right.$ and $\left.L_{3}=1\right)$, ( $L_{1}=2$ and $\left.L_{3}=1\right)$, and $\left(L_{1}=1\right.$ and $L_{3}=3$ ), all three give identical slopes achieving a diversity order of 4 as predicted

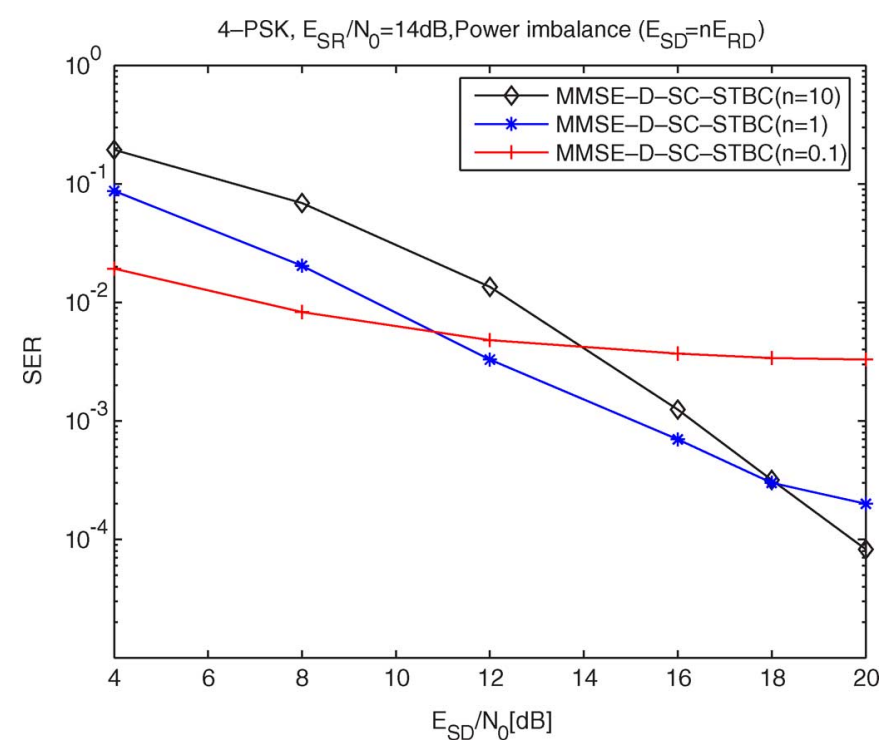

Fig. 8. SER performance of MMSE-D-SC-STBC scheme with power imbalance.

through (29)-(31). For the nonfading $R \rightarrow D$ link, we observe a diversity order of 4 as predicted by (23). We should emphasize here that the complexity of the MLSE receiver (given by the number of trellis states in the Viterbi algorithm) is adjusted according to the channel memory length for each scenario to extract the available multipath diversity. For instance, we use a total of $4^{3}$ states and $4^{4}$ states for $\left(L_{1}=1\right.$ and $\left.L_{3}=1\right)$ and ( $L_{1}=2$ and $L_{3}=1$ ), respectively.

Fig. 8 depicts the SER performance of D-SC-STBC scheme with power imbalance, i.e., $E_{S D} \neq E_{R D}$. We assume $E_{S D}=$ $n E_{R D}$ and compare the error rate performance for two different ratios, i.e., $n=0.1$ and 10. We also include the performance of perfect power control case, i.e., $n=1$ as a benchmark. We observe performance improvement in the low-to-intermediate SNR range for $n=0.1$, i.e., $E_{R D} \gg E_{S D}$ in comparison to the balanced case, i.e., $n=1$; however, this also results in an immediate error floor. On the other hand, larger values of $n$ result in performance degradation in the low-to-intermediate SNR range while it results in performance improvement over the perfect power control case for asymptotically high SNR values.

Fig. 9 depicts the SER performance of coded D-OFDMSTBC and compares it to that of D-TR-STBC. A 16-state 8-PSK Ungerboeck code with rate $=2 / 3$ and $E C L=3$ (labeled as U16) [28] is employed as an outer code. We assume $E_{S R} / N_{0}=35 \mathrm{~dB}$ and consider the following scenarios:

1) $L_{1}=L_{2}=L=1$ and nonfading $R \rightarrow D$ link, i.e., $L_{3}=0$ and $\mathbf{h}_{3}[0]=1$.

2) $L_{1}=L_{2}=L=1$ and frequency flat $R \rightarrow D$ link, i.e., $L_{3}=0$.

As this figure clearly demonstrates, coded D-OFDM-STBC has comparable performance to D-TR-STBC achieving the same diversity order. Specifically, D-OFDM-STBC achieves a diversity order of 4 , as predicted by (53), i.e. $2 \times \min (\lceil E C L / 2\rceil=2, L+1=2)=4$. For the second scenario, we observe a diversity order of 3 confirming (54), i.e. $\min (\lceil E C L / 2\rceil=2,2,1)+\min (\lceil E C L / 2\rceil=2,2)=3$. It 


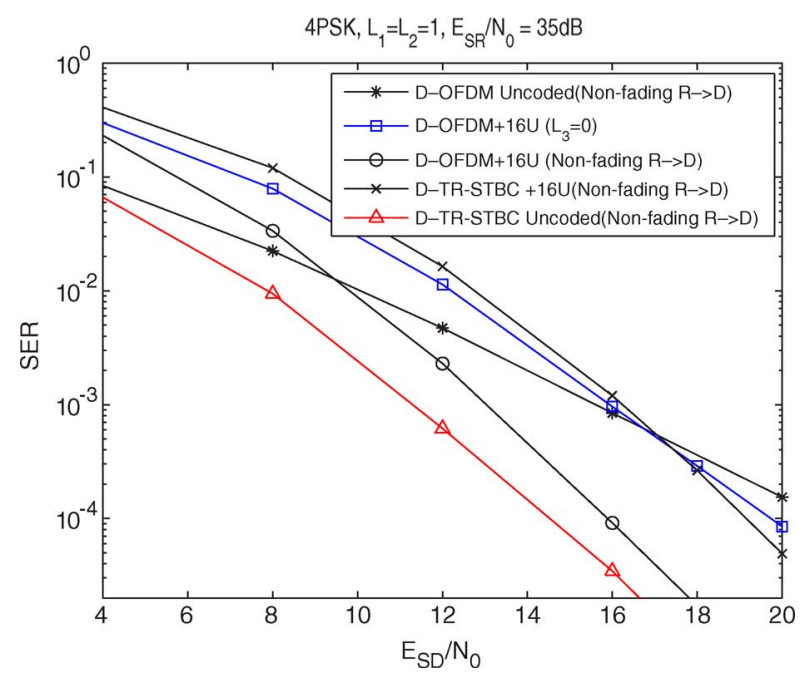

Fig. 9. SER performances of D-TR-STBC and D-STBC-OFDM schemes with outer TCM code.

is also interesting to note that the performance of the uncoded D-TR-STBC outperforms its coded version. This is a result of the fact that we assume separate decoding and equalization in our implementation. A similar observation has been reported in [35], [36] in the context of conventional TCM systems.

\section{CONCLUSION}

We have investigated the performance of three equalization schemes proposed originally for nondistributed STBC schemes within a cooperative user scenario, carefully exploiting the underlying orthogonality of distributed STBC. Assuming a single-relay scenario where $S \rightarrow R, R \rightarrow D$, and $S \rightarrow D$ links experience different channel delay spreads, we derived PEP expressions for D-TR-STBC, D-SC-STBC, and D-OFDM-STBC. Under the assumption of perfect power control for the relay terminal and high SNR for the underlying links, our performance analysis shows that D-TR-STBC and D-SC-STBC schemes yield identical performance achieving a maximum diversity order of $L_{1}+L_{2}+2$ for a nonfading $R \rightarrow D$ link and $L_{3}+L_{2}+2$ for a nonfading $S \rightarrow R$ link, where $L_{1}, L_{2}$, and $L_{3}$ are the channel memory lengths for $S \rightarrow R, S \rightarrow D$, and $R \rightarrow D$ links, respectively. For the general case where all underlying links experience frequency selectivity, these two schemes achieve a diversity order of $\min \left(L_{1}, L_{3}\right)+L_{2}+2$. Under the same assumptions, the uncoded D-OFDM-STBC scheme achieves only a maximum diversity order of two since it is not able to exploit the underlying multipath diversity. However, by combining D-OFDM-STBC scheme with interleaving and a judiciously-designed outer code, it is able to provide the same diversity of D-TR-STBC and D-SC-STBC. Extensive Monte Carlo simulation results were further presented to corroborate our diversity gain analysis and to provide detailed comparisons among the three candidate schemes.

\section{APPENDIX I}

DERIVATION OF (22)

In this section, we derive the PEP expression for D-TR-STBC scheme for nonfading $R \rightarrow D$ link and frequency-selective
$S \rightarrow R$ and $S \rightarrow D$ links. Defining the transmitted codeword vector and erroneously decoded codeword vector as $\mathbf{x}$ and $\hat{\mathbf{x}}$, respectively, the Chernoff bound on PEP is given as

$$
P\left(\mathbf{x}, \hat{\mathbf{x}} \mid \mathbf{h}_{1}, \mathbf{h}_{2}\right) \leq \exp \left(-\frac{d^{2}(\mathbf{x}, \hat{\mathbf{x}})}{4 N_{0}}\right)
$$

where $d^{2}(\mathbf{x}, \hat{\mathbf{x}})$ is earlier defined by (21).

By introducing $\boldsymbol{\chi}_{i}=\left(\boldsymbol{\chi}_{i}-\hat{\boldsymbol{\chi}}_{i}\right)\left(\boldsymbol{\chi}_{i}-\hat{\boldsymbol{\chi}}_{i}\right)^{\mathrm{H}}$ with dimensions of $\left(L_{i}+1\right) \times\left(L_{i}+1\right),(21)$ can be written as follows

$$
d^{2}(\mathbf{x}, \hat{\mathbf{x}})=\gamma_{1} \mathbf{h}_{1}^{\mathrm{T}} \boldsymbol{\chi}_{1}\left(\mathbf{h}_{1}^{\mathrm{T}}\right)^{\mathrm{H}}+\gamma_{2} \mathbf{h}_{2}^{\mathrm{T}} \boldsymbol{\chi}_{2}\left(\mathbf{h}_{2}^{\mathrm{T}}\right)^{\mathrm{H}}
$$

Further defining $\boldsymbol{\Omega}_{i}=\operatorname{diag}\left(\mathbf{v}_{i}\right), \boldsymbol{\mu}_{i}=\boldsymbol{\Omega}_{i}^{-1 / 2} \mathbf{h}_{i}$, and $\mathbf{A}_{i}=$ $\boldsymbol{\Omega}_{i}^{1 / 2} \boldsymbol{\chi}_{i} \boldsymbol{\Omega}_{i}^{1 / 2}, i=1,2$, we obtain

$$
d^{2}(\mathbf{x}, \hat{\mathbf{x}})=\gamma_{1} \boldsymbol{\mu}_{1}^{\mathrm{T}} \mathbf{A}_{1}\left(\boldsymbol{\mu}_{1}^{\mathrm{T}}\right)^{\mathrm{H}}+\gamma_{2} \boldsymbol{\mu}_{2}^{\mathrm{T}} \mathbf{A}_{2}\left(\boldsymbol{\mu}_{2}^{\mathrm{T}}\right)^{\mathrm{H}}
$$

where $\mathbf{A}_{i}, i=1,2$, are full rank due to zero padding [27]. Since $\mathbf{A}_{i}$ is Hermitian, there exists a unitary matrix $\mathbf{U}_{\mathbf{A}_{i}}$ such that $\mathbf{U}_{\mathbf{A}_{i}}^{\mathrm{H}} \mathbf{A}_{i} \mathbf{U}_{\mathbf{A}_{i}}=\boldsymbol{\Delta}_{i}$ where $\boldsymbol{\Delta}_{i}$ is a real diagonal matrix of size $\left(L_{i}+1\right) \times\left(L_{i}+1\right)$ with nonnegative entries. Noting that the diagonal elements of $\Delta_{i}$ are the eigenvalues of $\mathbf{A}_{i}$, (57) can be written as

$$
d^{2}(\mathbf{x}, \hat{\mathbf{x}})=\gamma_{1} \boldsymbol{\beta}_{1} \boldsymbol{\Delta}_{1} \boldsymbol{\beta}_{1}^{\mathrm{H}}+\gamma_{2} \boldsymbol{\beta}_{2} \boldsymbol{\Delta}_{2} \boldsymbol{\beta}_{2}^{\mathrm{H}}
$$

where $\boldsymbol{\beta}_{i}=\boldsymbol{\mu}_{i}^{\mathrm{T}} \mathbf{U}_{\mathbf{A}_{i}}, i=1,2$, is a zero-mean complex Gaussian vector with unit variance. Thus, we can express (58) as

$$
d^{2}(\mathbf{x}, \hat{\mathbf{x}})=\gamma_{1} \sum_{l_{1}=0}^{L_{1}} \boldsymbol{\lambda}_{1}\left(l_{1}\right)\left|\boldsymbol{\beta}_{1}\left(l_{1}\right)\right|^{2}+\gamma_{2} \sum_{l_{2}=0}^{L_{2}} \boldsymbol{\lambda}_{2}\left(l_{2}\right)\left|\boldsymbol{\beta}_{2}\left(l_{2}\right)\right|^{2}
$$

where $\boldsymbol{\lambda}_{i}\left(l_{i}\right)$ denotes the $l_{i}^{\text {th }}$ eigenvalue of $\boldsymbol{\Delta}_{i}$. Substituting (59) in (55) and averaging the resulting expression with respect to $\left|\boldsymbol{\beta}_{1}\left(l_{1}\right)\right|$ and $\left|\boldsymbol{\beta}_{2}\left(l_{2}\right)\right|$ which are Rayleigh distributed, we obtain the final PEP expression as in (22)

$$
\begin{aligned}
P(\mathbf{x}, \hat{\mathbf{x}}) \leq\left(1+\frac{\gamma_{1}}{4 N_{0}}\right)^{-\left(L_{1}+1\right)} & \left(1+\frac{\gamma_{2}}{4 N_{0}}\right)^{-\left(L_{2}+1\right)} \\
\times & \prod_{l_{1}=0}^{L_{1}} \frac{1}{\lambda_{1}\left(l_{1}\right)} \prod_{l_{2}=0}^{L_{2}} \frac{1}{\lambda_{2}\left(l_{2}\right)} .
\end{aligned}
$$

\section{APPENDIX II}

DERIVATION OF (29)-(31)

In this Appendix, we derive the PEP expression for the D-TRSTBC scheme assuming frequency-selective channels in $S \rightarrow$ $R, S \rightarrow D$, and $R \rightarrow D$ links. The Chernoff bound on PEP is given as

$$
P\left(\mathbf{x}, \hat{\mathbf{x}} \mid \mathbf{h}_{1}, \mathbf{h}_{2}, \mathbf{h}_{3}\right) \leq \exp \left(-\frac{d^{2}(\mathbf{x}, \hat{\mathbf{x}})}{4 N_{0}}\right)
$$

The Euclidean distance between $\mathbf{x}$ and $\hat{\mathbf{x}}$ can be written as

$$
d^{2}(\mathbf{x}, \hat{\mathbf{x}})=\gamma_{1}\left\|\mathbf{H}_{3} \mathbf{H}_{1} \mathbf{e}\right\|^{2}+\gamma_{2}\left\|\mathbf{H}_{2} \mathbf{e}\right\|^{2}
$$


where $\gamma_{1}=\gamma_{2}=E_{S D}$ and $\mathbf{e}=\mathbf{d}-\hat{\mathbf{d}}$. To simplify analysis, (62) can be approximated ${ }^{5}$ as

$$
\begin{aligned}
d^{2}(\mathbf{x}, \hat{\mathbf{x}}) & \approx \frac{\gamma_{1}}{N}\left\|\mathbf{H}_{3}\right\|^{2}\left\|\mathbf{H}_{1} \mathbf{e}\right\|^{2}+\gamma_{2}\left\|\mathbf{H}_{2} \mathbf{e}\right\|^{2} \\
& \approx \frac{\gamma_{1}}{N}\left\|\mathbf{H}_{1}\right\|^{2}\left\|\mathbf{H}_{3} \mathbf{e}\right\|^{2}+\gamma_{2}\left\|\mathbf{H}_{2} \mathbf{e}\right\|^{2}
\end{aligned}
$$

where $N$ is the frame length. Noting that $\left\|\mathbf{H}_{i}\right\|^{2}=$ $N \sum_{l_{i=0}}^{L_{i}}\left|\mathbf{h}_{i}\left(l_{i}\right)\right|^{2}, i=1,2$, we can further write (63) as

$$
\begin{aligned}
d^{2}(\mathbf{x}, \hat{\mathbf{x}}) & \approx \gamma_{1} \sum_{l 3=0}^{L_{3}}\left|\mathbf{h}_{3}\left(l_{3}\right)\right|^{2}\left\|\mathbf{H}_{1} \mathbf{e}\right\|^{2}+\gamma_{2}\left\|\mathbf{H}_{2} \mathbf{e}\right\|^{2} \\
& \approx \gamma_{1} \sum_{l_{1}=0}^{L_{1}}\left|\mathbf{h}_{1}\left(l_{1}\right)\right|^{2}\left\|\mathbf{H}_{3} \mathbf{e}\right\|^{2}+\gamma_{2}\left\|\mathbf{H}_{2} \mathbf{e}\right\|^{2}
\end{aligned}
$$

Following similar steps in Appendix I, we can write (64) as

$$
\begin{aligned}
d^{2}(\mathbf{x}, \hat{\mathbf{x}}) \approx \gamma_{1} \sum_{l_{3=0}}^{L_{3}}\left|\mathbf{h}_{3}\left(l_{3}\right)\right|^{2} \sum_{l_{1}=0}^{L_{1}} \boldsymbol{\lambda}_{1}\left(l_{1}\right)\left|\boldsymbol{\beta}_{1}\left(l_{1}\right)\right|^{2} \\
+\gamma_{2} \sum_{l_{2}=0}^{L_{2}} \boldsymbol{\lambda}_{2}\left(l_{2}\right)\left|\boldsymbol{\beta}_{2}\left(l_{2}\right)\right|^{2}
\end{aligned}
$$

or

$$
\begin{aligned}
d^{2}(\mathbf{x}, \hat{\mathbf{x}}) \approx \gamma_{1} \sum_{l_{1=0}}^{L_{1}}\left|\mathbf{h}_{1}\left(l_{1}\right)\right|^{2} \sum_{l 3=0}^{L_{3}} & \boldsymbol{\lambda}_{1}\left(l_{3}\right)\left|\boldsymbol{\beta}_{1}\left(l_{3}\right)\right|^{2} \\
& +\gamma_{2} \sum_{\boldsymbol{l}_{2}=0}^{L_{2}} \boldsymbol{\lambda}_{2}\left(l_{2}\right)\left|\boldsymbol{\beta}_{2}\left(l_{2}\right)\right|^{2}
\end{aligned}
$$

where $\boldsymbol{\lambda}_{i}\left(l_{i}\right), i=1,2$ denote the $l^{\text {th }}$ eigenvalue of codeword difference matrixes and $\boldsymbol{\beta}_{i}$ are zero-mean complex Gaussian vectors with unit variance. In the following, we will derive the PEP expressions under three different scenarios:

Case 1: $L_{3}>L_{1}$ : First, we define the random variables $Y_{2}=\sum_{l_{2}=0}^{L_{2}} \boldsymbol{\lambda}_{2}\left(l_{2}\right)\left|\boldsymbol{\beta}_{2}\left(l_{2}\right)\right|^{2}$ and $Y_{1}=X_{1} X_{2}$ with $X_{1}=\sum_{l_{3}=0}^{L_{3}}\left|\mathbf{h}_{3}\left(l_{3}\right)\right|^{2}$ and $X_{2}=\sum_{l_{1}=0}^{L_{1}} \boldsymbol{\lambda}_{1}\left(l_{1}\right)\left|\boldsymbol{\beta}_{1}\left(l_{1}\right)\right|^{2}$. Substituting (65) in (61) and averaging the resulting expression with respect to $Y_{1}$ and $Y_{2}$, we obtain

$$
\begin{aligned}
P(\mathbf{x}, \hat{\mathbf{x}}) & \leq E_{Y_{1}}\left[\exp \left(-\frac{E_{S D}}{4 N_{0}} Y_{1}\right)\right] E_{Y_{2}}\left[\exp \left(-\frac{E_{S D}}{4 N_{0}} Y_{2}\right)\right] \\
& =\left.\Phi_{Y_{1}}(\omega)\right|_{j \omega=-\frac{E_{S D}}{4 N_{0}}} \times\left.\Phi_{Y_{2}}(\omega)\right|_{j \omega=-\frac{E_{S D}}{4 N_{0}}}
\end{aligned}
$$

where $\Phi_{Y_{1}}(\omega)$ and $\Phi_{Y_{2}}(\omega)$ are the characteristic functions of $Y_{1}$ and $Y_{2}$, respectively. Since the entries of $\left|\boldsymbol{\beta}_{2}\right|$ are Rayleigh distributed, $\Phi_{Y_{2}}(\omega)$ is given as [38]

$$
\left.\Phi_{Y_{2}}(\omega)\right|_{j \omega=-\frac{E_{S D}}{4 N_{0}}}=\prod_{l_{2}=0}^{L_{2}}\left(1+\frac{E_{S D}}{4 N_{0}} \lambda_{2}\left(l_{2}\right)\right)^{-1}
$$

${ }^{5}$ Through a Monte-Carlo simulation experiment, we observed that the mean square error (MSE) difference between (62) and (63) is only 0.3 at $E_{S D} / N_{0}=4 \mathrm{~dB}$. The approximate expression converges to the exact one as early as $E_{S D} / N_{0}=6 \mathrm{~dB}$. Therefore, this approximation can be used safely for any practical purpose.
$\Phi_{Y_{1}}(\omega)$ can be evaluated as [39]

$$
\Phi_{Y 1}(\omega)=\int_{0}^{\infty} f_{X_{1}}\left(x_{1}\right) \Phi_{X_{2}}\left(\omega x_{1}\right) d x_{1}
$$

where $f_{X_{1}}\left(x_{1}\right), x_{1} \geq 0$ is the probability density function (pdf) of $X_{1}$ and $\Phi_{X_{2}}\left(\omega x_{1}\right)$ is the characteristic function of $X_{2}$. In our case, the entries of $\mathbf{h}_{3}$ are modeled as zero-mean complex Gaussian random variables with variance $1 / 2\left(L_{3}+1\right)$ per-dimension (i.e. uniform power profile). Therefore, $X_{2}$ is a chisquared random variable with $2\left(L_{3}+1\right)$ degrees of freedom [40]

$$
f_{X_{1}}\left(x_{1}\right)=\frac{\left(L_{3}+1\right)^{L_{3}+1}}{\Gamma\left(L_{3}+1\right)} x_{1}^{L_{3}} e^{-x_{1}\left(L_{3}+1\right)} .
$$

Substituting (70) in (69) and also noting $\Phi_{X_{2}}\left(\omega x_{1}\right)=$ $\prod_{l_{1}=0}^{L_{1}} 1 /\left(1-j \omega x_{1} \lambda_{1}\left(l_{1}\right)\right)$ [38], (69) can be written as

$$
\begin{gathered}
\left.\Phi_{Y_{1}}(\omega)\right|_{j \omega=-\frac{E_{S D}}{4 N_{0}}}=\frac{\left(L_{3}+1\right)^{L_{3}+1}}{\Gamma\left(L_{3}+1\right)}\left(\frac{E_{S D}}{4 N_{0}}\right)^{-\left(L_{1}+1\right)} \\
\times \prod_{l_{1}=0}^{L_{1}}\left(\boldsymbol{\lambda}_{1}\left(l_{1}\right)\right)^{-1} \int_{0}^{\infty} \frac{x_{1}^{L_{3}} e^{-x_{1}\left(L_{3}+1\right)}}{\prod_{l_{1}}^{L_{1}}\left(\frac{1}{\boldsymbol{\lambda}_{1}\left(l_{1}\right) E_{S D} / 4 N_{0}}+x_{1}\right)} d x_{1} .
\end{gathered}
$$

Assuming high SNR, i.e. $E_{S D} / 4 N_{0} \gg 1$, (71) yields

$$
\begin{aligned}
\left.\Phi_{Y_{1}}(\omega)\right|_{j \omega=} & =\frac{E_{S D}}{4 N_{0}}=\frac{\left(L_{3}+1\right)^{L_{3}+1}}{\Gamma\left(L_{3}+1\right)}\left(\frac{E_{S D}}{4 N_{0}}\right)^{-\left(L_{1}+1\right)} \\
& \times \prod_{l_{1}=0}^{L_{1}}\left(\boldsymbol{\lambda}_{1}\left(l_{1}\right)\right)^{-1} \int_{0}^{\infty} x_{1}^{L_{3}-L_{1}} e^{-x_{1}\left(L_{3}+1\right)} d x_{1} .
\end{aligned}
$$

Using the integral form given by [25, p.382, 3.351.3], we obtain

$$
\begin{aligned}
\left.\Phi_{Y_{1}}(\omega)\right|_{j \omega=-\frac{E_{S D}}{4 N_{0}}} & =\left(L_{3}+1\right)^{L_{1}} \frac{\Gamma\left(L_{3}-L_{1}+1\right)}{\Gamma\left(L_{3}+1\right)} \\
& \times\left(\frac{E_{S D}}{4 N_{0}}\right)^{-\left(L_{1}+1\right)} \prod_{l_{1}=0}^{L_{1}}\left(\boldsymbol{\lambda}_{1}\left(l_{1}\right)\right)^{-1} .
\end{aligned}
$$

Substituting (73) and (68) in (67), we find the final PEP expression as

$$
\begin{aligned}
P(\mathbf{x}, \hat{\mathbf{x}}) \leq\left(\frac{E_{S D}}{4 N_{0}}\right)^{-\left(L_{1}+L_{2}+2\right)}\left(L_{3}+1\right)^{L_{1}} \frac{\Gamma\left(L_{3}-L_{1}+1\right)}{\Gamma\left(L_{3}+1\right)} \\
\times \prod_{l_{1}=0}^{L_{1}}\left(\boldsymbol{\lambda}_{1}\left(l_{1}\right)\right)^{-1} \prod_{l_{2}=0}^{L_{2}}\left(\boldsymbol{\lambda}_{2}\left(l_{2}\right)\right)^{-1} \cdot
\end{aligned}
$$

Case 2: $L_{1}>L_{3}$ : Using (66) and noting that this case is similar to Case 1 with $L_{1}$ and $L_{3}$ now interchanged, the PEP expression is given as

$$
\begin{aligned}
P(\mathbf{x}, \hat{\mathbf{x}}) \leq\left(\frac{E_{S D}}{4 N_{0}}\right)^{-\left(L_{3}+L_{2}+2\right)}\left(L_{1}+1\right)^{L_{3}} \frac{\Gamma\left(L_{1}-L_{3}+1\right)}{\Gamma\left(L_{1}+1\right)} \\
\times \prod_{l_{3}=0}^{L_{3}}\left(\boldsymbol{\lambda}_{1}\left(l_{3}\right)\right)^{-1} \prod_{l_{2}=0}^{L_{2}}\left(\boldsymbol{\lambda}_{2}\left(l_{2}\right)\right)^{-1}
\end{aligned}
$$


Case 3: $L_{3}=L_{1}$ : Following the same argument in Case 1 and using partial fraction expansion, (71) can be rewritten as

$$
\begin{aligned}
\left.\Phi_{Y_{1}}(\omega)\right|_{j \omega=-\frac{E_{S D}}{4 N_{0}}}= & \frac{\left(L_{3}+1\right)^{L_{3}+1}}{\Gamma\left(L_{3}+1\right)} \sum_{l_{1}=1}^{L_{1}+1} \frac{p_{l_{1}}}{\lambda_{1}\left(l_{1}\right) E_{S D} / 4 N_{0}} \\
& \times \int_{0}^{\infty} \frac{x_{1}^{L_{3}} e^{-x_{1}\left(L_{3}+1\right)}}{\left(\frac{1}{\lambda_{1}\left(l_{1}\right) E_{S D} / 4 N_{0}}+x_{1}\right)} d x_{1}
\end{aligned}
$$

where $p_{\boldsymbol{l}_{1}}=\prod_{l=0, l \neq l_{1}}^{L_{1}}\left(\boldsymbol{\lambda}_{1}\left(l_{1}\right) / \boldsymbol{\lambda}_{1}\left(l_{1}\right)-\boldsymbol{\lambda}_{1}(l)\right)$. Using the integral form given by $[25, \mathrm{p} .338,3.353 .5 .7]$ and after some mathematical manipulation, we obtain

$$
\begin{aligned}
\left.\Phi_{Y_{1}}(\omega)\right|_{j \omega=-\frac{E_{S D}}{4 N_{0}}} & \frac{\left(L_{3}+1\right)^{L_{3}+1}}{\Gamma\left(L_{3}+1\right)}(-1)^{L_{3}-1}\left(\frac{E_{S D}}{4 N_{0}}\right)^{-\left(L_{3}+1\right)} \\
& \times \sum_{l_{1}=1}^{L_{1}+1} \frac{p_{l_{1}}}{\left(\boldsymbol{\lambda}_{1}\left(l_{1}\right)\right)^{L_{3}+1}} e^{\beta} E_{i}(-\beta)+C \\
C= & \frac{\left(L_{3}+1\right)^{L_{3}+1}}{\Gamma\left(L_{3}+1\right)} \sum_{l_{1}=1}^{L_{1}+1} \sum_{k=1}^{L_{3}}(k-1) !(-1)^{L_{3}-k}\left(L_{3}+1\right)^{-k} \\
& \times \frac{p_{l_{1}}}{\left(\boldsymbol{\lambda}_{1}\left(l_{1}\right)\right)^{L_{3}+1-k}}\left(\frac{E_{S D}}{4 N_{0}}\right)^{-\left(L_{3}+1-k\right)} .
\end{aligned}
$$

Using the fact $\sum_{l_{1}=1}^{L_{1}+1} p_{l_{1}} / \lambda_{1}^{r}\left(l_{1}\right)=0,1 \leq r \leq L_{1}$ and noting that $L_{1}=L_{3}, C$ becomes zero. Substituting (77) and (68) in (67) and assuming high SNR, we find the final PEP expression given as in (31).

APPENDIX III

DERIVATION OF (50)

Here, we derive the PEP expression for the D-OFDM-STBC scheme assuming a nonfading $R \rightarrow D$ link. For this scheme, the distance metric $d^{2}(\mathbf{x}, \hat{\mathbf{x}})$ is given by

$$
\begin{aligned}
d^{2}(\mathbf{x}, \hat{\mathbf{x}})=\gamma_{1}\left\|\left(\operatorname{diag}(\mathbf{x}-\hat{\mathbf{x}}) \mathbf{f}_{1}\right) \mathbf{h}_{1}\right\|^{2} & \\
& +\gamma_{2}\left\|\left(\operatorname{diag}(\mathbf{x}-\hat{\mathbf{x}}) \mathbf{f}_{2}\right) \mathbf{h}_{2}\right\|^{2}
\end{aligned}
$$

where we define $\mathbf{f}_{i}=\exp (-j 2 \pi(p-1)(q-1) / N), p=$ $1,2, \ldots, N, q=1, \ldots L_{i}+1$. This can be further simplified as

$$
d^{2}(\mathbf{x}, \hat{\mathbf{x}})=\gamma_{1} \boldsymbol{\varepsilon}_{1}^{\mathrm{H}} \boldsymbol{\Sigma}_{1} \varepsilon_{1}+\gamma_{2} \varepsilon_{2}^{\mathrm{H}} \boldsymbol{\Sigma}_{2} \boldsymbol{\varepsilon}_{2}
$$

where

$$
\boldsymbol{\Sigma}_{i}=\mathbf{U}_{i}^{\mathrm{H}} \boldsymbol{\Omega}_{i}^{1 / 2}\left(\operatorname{diag}(\mathbf{x}-\hat{\mathbf{x}}) \mathbf{f}_{i}\right)^{\mathrm{H}}\left(\operatorname{diag}(\mathbf{x}-\hat{\mathbf{x}}) \mathbf{f}_{i}\right) \boldsymbol{\Omega}_{i}^{1 / 2} \mathbf{U}_{i},
$$

$\varepsilon_{i}=\mathbf{U}_{i}^{\mathrm{H}} \boldsymbol{\Omega}_{i}^{-1 / 2} \mathbf{h}_{i}$, and $\mathbf{U}_{i}$ is a unitary matrix, $i=1,2$. Note that $\Sigma_{i}$ is a rank-one Hermitian matrix of size $\left(L_{i}+1\right) \times$
$\left(L_{i}+1\right)$. Substituting (79) in (55), we follow similar steps as in Appendix I to obtain the final form for PEP

$$
P(\mathbf{x}, \hat{\mathbf{x}}) \leq\left(\frac{E_{S D}}{4 N_{0}}\right)^{-2} \frac{1}{\lambda_{\Sigma_{1}}} \frac{1}{\lambda_{\Sigma_{2}}}
$$

where $\lambda_{\Sigma_{1}}, \lambda_{\Sigma_{2}}$ are the eigenvalues of $\boldsymbol{\Sigma}_{1}$ and $\boldsymbol{\Sigma}_{2}$, respectively.

\section{APPENDIX IV}

DERIVATION OF (51)

Here, we derive the PEP expression for the D-OFDM-STBC scheme given that all underlying links are frequency-selective. For this scheme, the distance metric $d^{2}(\mathbf{x}, \hat{\mathbf{x}})$ is given by

$$
\begin{aligned}
d^{2}(\mathbf{x}, \hat{\mathbf{x}})=\gamma_{1}\left\|\left(\operatorname{diag}(\mathbf{x}-\hat{\mathbf{x}}) \mathbf{f}_{1}\right) \mathbf{h}_{1} \mathbf{f}_{3} \mathbf{h}_{3}\right\|^{2} \\
+\gamma_{2}\left\|\left(\operatorname{diag}(\mathbf{x}-\hat{\mathbf{x}}) \mathbf{f}_{2}\right) \mathbf{h}_{2}\right\|^{2} .
\end{aligned}
$$

Using similar arguments of Appendix II, we can find an approximation for the distance metric as

$$
d^{2}(\mathbf{x}, \hat{\mathbf{x}}) \approx \gamma_{1} \sum_{l_{3}=0}^{L_{3}}\left|\mathbf{h}_{3}\left(l_{3}\right)\right|^{2} \varepsilon_{1}^{\mathrm{H}} \boldsymbol{\Sigma}_{1} \varepsilon_{1}+\gamma_{2} \varepsilon_{2}^{\mathrm{H}} \boldsymbol{\Sigma}_{2} \varepsilon_{2} .
$$

Substituting (82) in (61) and following the steps in detailed in Appendix II, we obtain

$$
P(\mathbf{x}, \hat{\mathbf{x}}) \leq\left(\frac{E_{S D}}{4 N_{0}}\right)^{-2} \frac{\Gamma\left(L_{3}\right)\left(L_{3}+1\right)}{\Gamma\left(L_{3}+1\right)} \frac{1}{\lambda_{\Sigma_{1}}} \frac{1}{\lambda_{\Sigma_{2}}}
$$

where $\lambda_{\Sigma_{1}}, \lambda_{\Sigma_{2}}$ are the eigenvalues of $\Sigma_{1}$ and $\Sigma_{2}$, respectively.

\section{APPENDIX V}

DERIVATION OF (52)

Here, we derive the PEP expression for trellis-coded D-OFDM-STBC scheme. Instead of the uncoded data in the original D-OFDM-STBC scheme, the TCM encoder outputs are interleaved and then precoded by the IFFT matrix $\mathbf{Q}^{H}$. Denote $\left.\mathbf{x}=\left[x_{1}[0] x_{2}[0] x_{1}[1] x_{2}\right][1] \ldots x_{1}[N-1] x_{2}[N-1]\right]$ as the output of the TCM encoder. Furthermore, let the transmitted symbols be $\mathbf{x}_{1}=\left[x_{1}[0] x_{1}[1] \ldots x_{1}[N-1]\right]$ and $\left.\mathbf{x}_{2}=\left[x_{2}[0] x_{2}\right][1] \ldots x_{2}[N-1]\right]$. For this scheme, assuming nonfading $R \rightarrow D$ link, we have

$$
d^{2}(\mathbf{x}, \hat{\mathbf{x}})=\gamma_{1} \varepsilon_{1}^{\mathrm{H}} \boldsymbol{\Theta}_{1} \varepsilon_{1}+\gamma_{2} \varepsilon_{2}^{\mathrm{H}} \boldsymbol{\Theta}_{2} \varepsilon_{2}
$$

where $\boldsymbol{\Theta}_{i}=\mathbf{U}_{i}^{\mathrm{H}} \boldsymbol{\Omega}_{i}^{1 / 2} \mathbf{f}_{i}^{\mathrm{H}} \mathbf{P f}_{i} \boldsymbol{\Omega}_{i}^{1 / 2} \mathbf{U}_{i}$ and $\mathbf{P}=\operatorname{diag}\left(\mid \mathbf{x}_{1}-\right.$ $\left.\left.\hat{\mathbf{x}}_{1}\right|^{2}+\left|\mathbf{x}_{2}-\hat{\mathbf{x}}_{2}\right|^{2}\right)$. We further define $r_{i}$ and $\lambda_{\Theta_{i}}\left(j_{i}\right), j_{i}=$ $1, \ldots, r_{i}$ for $i=1,2$, as the rank and the eigenvalues of $\boldsymbol{\Theta}_{i}$, 
respectively. Replacing (84) in (55) and following similar steps as in Appendix I, we obtain the final PEP as

$P(\mathbf{x}, \hat{\mathbf{x}}) \leq\left(\frac{E_{S D}}{4 N_{0}}\right)^{-\beta} \times \prod_{j_{1}=1}^{r_{1}}\left(\boldsymbol{\lambda}_{\Theta_{1}}\left(j_{1}\right)\right)^{-1} \prod_{j_{2}=1}^{r_{2}}\left(\lambda_{\Theta_{2}}\left(j_{2}\right)\right)^{-1}$

where $\beta$ is defined as $\beta=r_{1}+r_{2}$.

\section{REFERENCES}

[1] E. Lindskog and A. Paulraj, "A transmit diversity scheme for channels with intersymbol interference," in Proc. Int. Conf. Commun., New Orleans, LA, Jun. 2002.

[2] N. Al-Dhahir, "Single carrier frequency domain equalization for space time block coded transmissions over frequency selective fading channels," IEEE Commun. Lett., vol. 5, no. 7, pp. 304-306, Jul. 2001.

[3] Z. Liu, G. B. Giannakis, A. Scaglione, and S. Barbarossa, "Block precoding and transmit-antenna diversity for decoding and equalization of unknown multipath channels," in Proc. 33rd Asilomar Conf. Signals, Syst., Comput., Pacific Grove, CA, Nov. 1999.

[4] J. G. Foschini, "Layered space-time architecture for wireless communication in a fading environment when using multi element antennas," Bell Labs Tech. J., vol. 2, pp. 41-59, Autumn, 1996.

[5] V. Tarokh, N. Seshadri, and A. R. Calderbank, "Space-time codes for high data rate wireless communication: performance criterion and code construction," IEEE Trans. Inf. Theory, vol. 44, no. 2, pp. 744-765, Mar. 1998.

[6] V. Tarokh, H. J. Jafarkhani, and A. R. Calderbank, "Space-time block codes from orthogonal designs," IEEE Trans. Inf. Theory, vol. 45, no. 5, pp. 1456-1467, Jul. 1999.

[7] S. M. Alamouti, "A simple transmit diversity technique for wireless communications," IEEE J. Sel. Areas Commun., vol. 16, no. 8, pp. 1451-1458, Oct. 1998.

[8] A. Sendonaris, E. Erkip, and B. Aazhang, "User cooperation diversity. Part I. System description," IEEE Trans. Commun., vol. 51, no. 11, pp. 1927-1938, Nov. 2003.

[9] —-, "User cooperation diversity. Part II. Implementation aspects and performance analysis," IEEE Trans. Commun., vol. 51, no. 11, pp. 1939-1948, Nov. 2003.

[10] J. N. Laneman and G. W. Wornell, "Distributed space-time-coded protocols for exploiting cooperative diversity in wireless networks," IEEE Trans. Inf. Theory, vol. 49, no. 10, pp. 2415-2425, Oct. 2003.

[11] M. Janani, A. Hedayat, T. E. Hunter, and A. Nosratinia, "Coded cooperation in wireless communications: space-time transmission and iterative decoding," IEEE Trans. Signal Process., vol. 52, no. 2, pp. 362-371, Feb. 2004

[12] R. U. Nabar, H. Boelcskei, and F. W. Kneubhueler, "Fading relay channels: performance limits and space-time signal design," IEEE J. Sel. Areas Commun., vol. 22, pp. 1099-1109, Aug. 2004.

[13] R. U. Nabar and H. Boelcskei, "Space-time signal design for fading relay channels," in IEEE GLOBECOM, San Francisco, CA, Dec. 2003.

[14] A. F. Naguib and N. Seshadri, "MLSE and equalization of space-time coded signals," in IEEE Vehicular Technology Conf. (VTC'00)-Spring, May 2000.

[15] N. Al-Dhahir, "Overview and comparison of equalization schemes for space-time coded signals with application to EDGE," IEEE Trans. Signal Process., vol. 50, no. 10, Oct. 2002.

[16] N. Al-Dhahir, M. Uysal, and C. N. Georghiades, "Three space-time block coding schemes for frequency-selective fading channels with application to EDGE," in Proc. IEEE Vehicular Technology Conf. (VTC'01)—Fall, Oct. 2001, pp. 1834-1838.

[17] H. Mheidat, M. Uysal, and N. Al-Dhahir, "Comparative analysis of equalization techniques for STBC with application to EDGE," in Proc. IEEE Vehicular Technology Conf. (VTC'04)—Spring, Milan, Italy, May 2004.

[18] H. Mheidat and M. Uysal, "Equalization techniques for space-time coded cooperative systems," in Proc. IEEE Vehicular Technology Conf. (VTC'04)—Fall, Los Angeles, CA, Sep. 2004.

[19] S. Yatawatta and A. P. Petropulu, "A multiuser OFDM system with user cooperation," in 38th Asilomar Conf. Signals, Syst., Comput, Pacific Grove, CA, Nov. 2004.

[20] S. Barbarossa and G. Scutari, "Distributed space-time coding for multihop networks communications," in IEEE Int. Conf. Communications (ICC), Paris, France, Jun. 2004.
[21] P. A. Anghel and M. Kaveh, "Exact symbol error probability of a cooperative network in a Rayleigh-fading environment," IEEE Trans. Wireless Commun., vol. 3, no. 5, pp. 1416-1421, Sep. 2004.

[22] _ - "Relay assisted uplink communication over frequency-selective channels," in IEEE Int. Workshop Signal Processing Advances for Wireless Communications (SPAWC) 2003, New York, Jun. 15-18, 2003.

[23] S. Zhou and G. B. Giannakis, "Single-carrier space-time block coded transmissions over frequency-selective fading channels," IEEE Trans. Inf. Theory, vol. 49, no. 1, pp. 164-179, Jan. 2003.

[24] G. Forney, Jr., "Maximum-likelihood sequence estimation of digital sequence in the presence of intersymbol interference," IEEE Trans. Inf. Theory, vol. 18, pp. 363-378, May 1972.

[25] I. S. Gradshteyn and I. M. Ryzhik, Table of Integrals, Series and Products. New York: Academic, 2000.

[26] H. Sari, G. Karam, and I. Jeanclaude, "Transmission techniques for digital terrestrial TV broadcasting," IEEE Commun. Mag., no. 2, Feb. 1995.

[27] S. Zhou and G. B. Giannakis, "Space-time coding with maximum diversity gains over frequency-selective fading channels," IEEE Signal Process. Lett., vol. 8, no. 10, pp. 269-272, Oct. 2001.

[28] E. Biglieri, D. Divsalar, M. K. Simon, and P. J. McLane, Introduction to Trellis-Coded Modulation With Applications. New York: Macmillan, 1991.

[29] S. M. Alamouti, V. Tarokh, and P. Poon, "Trellis coded modulation and transmit diversity: design criteria and performance evaluation," in IEEE Int. Conf. Universal Personal Communications (ICUPC), Oct. 1998, pp. 703-707.

[30] M. Uysal and C. N. Georghiades, "Analysis of concatenated trellis coded STBC schemes over rician fading channels," in 39th Allerton Conf. Commun., Control Computing, Monticello, CA, Oct. 2001.

[31] Y. Gong and K. B. Letaief, "Concatenated space-time block coding with Trellis coded modulation in fading channels," IEEE Trans. Wireless Commun., vol. 1, no. 4, pp. 580-590, Oct. 2002.

[32] M. Uysal and C. N. Georghiades, "Upper bounds on the BER performance of MTCM-STBC schemes over shadowed Rician fading channels," EURASIP J. Appl. Signal Process., vol. 4, no. 9, pp. 1238-1245, Aug. 2004.

[33] Y. Gong and K. B. Letaief, "An efficient space-frequency coded OFDM system for broadband wireless communications," IEEE Trans. Commun., vol. 51, no. 12, pp. 2019-2029, December 2003.

[34] Z. Liu, Y. Xin, and G. B. Giannakis, "Space-time-frequency coded OFDM over frequency-selective fading channels," IEEE Trans. Signal Process., vol. 50, no. 10, pp. 2465-2476, Oct. 2002.

[35] K. A. Hamied and G. L. Stuber, "Performance of trellis-coded modulation for equalized multipath fading ISI channels," IEEE Trans. Veh. Technol., vol. 44, no. 1, pp. 50-58, Feb. 1995.

[36] E. Baccarelli and S. Galli, "New results about analysis and design of TCM for ISI channels and combined equalization/decoding," IEEE Trans. Commun., vol. 46, no. 4, pp. 417-420, Apr. 1998.

[37] O. Canpolat and M. Uysal, "Super-orthogonal space-time trellis coded cooperative diversity schemes," in IEEE Vehicular Technology Conf. (VTCVTC'04)—Fall, Los Angeles, CA, Sep. 2004

[38] M. K. Simon and M. S. Alouini, Digital Communication Over Fading Channels: A Unified Approach to Performance Analysis. New York: Wiley-Intersci., 2000.

[39] A. Stuart and J. K. Ord, Kendall's Advanced Theory of Statistics, 5th ed. London, U.K.: Griffin, 1987

[40] J. G. Proakis, Digital Communications, 3rd ed. New York: McGrawHill, 1995.

[41] C. Schlegel, "Error probability for multibeam Rayleigh channels," IEEE Trans. Commun., vol. 44, pp. 290-293, Mar. 1996.

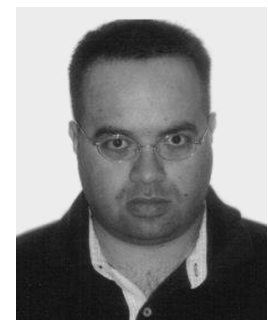

Hakam Mheidat (S'05-M'06) received the B.S degree from Yarmouk University, Jordan, and the M.S. degree from the University of Wisconsin, Milwaukee, in 1995 and 1999, respectively, both in electrical engineering.

Since January 2003, he has been with the Department of Electrical and Computer Engineering, University of Waterloo, Waterloo, Canada, working towards the Ph.D. degree. His current research focuses on equalization, multicarrier communication techniques, and physical layer aspects of wideband cooperative networks. 


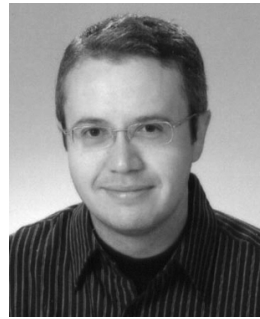

Murat Uysal (S'98-M'02) was born in Istanbul, Turkey, in 1973. He received the B.Sc. and the M.Sc. degrees in electronics and communication engineering from Istanbul Technical University, in 1995 and 1998, respectively, and the Ph.D. degree in electrical engineering from Texas A\&M University, College Station, in 2001.

Since April 2002, he has been with the Department of Electrical and Computer Engineering, University of Waterloo, Waterloo, Canada, as an Assistant Professor. His general research interests lie in communications theory and signal processing for communications with special emphasis on wireless applications. Specific research areas include space-time coding, MIMO techniques, performance analysis over fading channels, and cooperative communications.

Dr. Uysal is an Editor for the IEEE TRANSACTIONS ON WIRELESS COMMUNICATIONS and an Associate Editor for the IEEE COMMUNICATIONS LETTERS. He also served as a Guest Co-Editor for the Wiley Journal on Wireless Communications and Mobile Computing's Special Issue on MIMO Communications. He is currently serving as the Co-Chair of the IEEE ICC'07 Communication Theory Symposium.

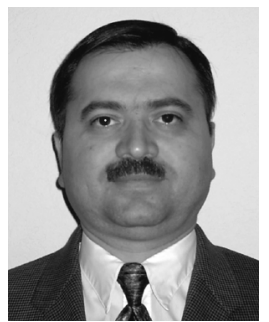

Naofal Al-Dhahir (M'90-SM'98) received the M.S. and $\mathrm{Ph} . \mathrm{D}$. degrees from Stanford University, Stanford, CA, in 1990 and 1994, respectively, in electrical engineering.

He was an Instructor at Stanford University in 1993. From August 1994 to July 1999, he was a member of Technical Staff at GE R\&D Center, NY, where he worked on various aspects of satellite communication systems design and antijam GPS receivers. From August 1999 to July 2003, he was a principal member of Technical Staff at AT\&T Shannon Laboratory, Florham Park, NJ, where he worked on space-time coding and signal processing. Currently, he is an Associate Professor with the University of Texas at Dallas. His current research interests include space-time coding and signal processing, OFDM, wireless networks, and digital subscriber line technology. He has authored more than 150 journal and conference papers and holds 16 U.S. patents. He is coauthor of the book Doppler Applications for LEO Satellite Systems (New York: Springer, 2002).

Dr. Al-Dhahir is a member of the IEEE SP4COM and SPTM technical committees. He served as Editor for the IEEE TRANSACTIONS ON Signal PROCESSING and the IEEE COMMUNICATIONS LETTERS. He is currently an Editor for the IEEE TRANSACTIONS ON COMMUNICATIONS. He served as co-chair of the Communication Theory Symposium at IEEE GLOBECOM'04. $\mathrm{He}$ is corecipient of the IEEE VTC Fall'05 Best Paper award, the 2005 IEEE Signal Processing Society Young Author Best Paper award, and the 2006 IEEE Donald G. Fink Best Paper award. 